\title{
Genomic and transcriptome analysis revealing an oncogenic functional module in meningiomas
}

\author{
Xiao Chang, Ph.D., ${ }^{1}$ Lingling Shi, Ph.D., ${ }^{2}$ Fan Gao, Ph.D., ${ }^{1}$ Jonathan Russin, M.D., ${ }^{3}$ \\ Liyun Zeng, Ph.D., ${ }^{1}$ Shuhan He, B.S., ${ }^{3}$ Thomas C. Chen, M.D., ${ }^{3}$ \\ Steven L. Giannotta, M.D. ${ }^{3}$ Daniel J. Weisenberger, Ph.D., ${ }^{4}$ Gabriel Zada, M.D., ${ }^{3}$ \\ Kai Wang, Ph.D., ${ }^{1,5,6}$ and William J. Mack, M.D., \\ ${ }^{1}$ Zilkha Neurogenetic Institute, Keck School of Medicine, University of Southern California, Los Angeles, \\ California; ${ }^{2}$ GHM Institute of CNS Regeneration, Jinan University, Guangzhou, China; ${ }^{3}$ Department of \\ Neurosurgery, Keck School of Medicine, University of Southern California, Los Angeles, California; ${ }^{4}$ USC \\ Epigenome Center, Keck School of Medicine, University of Southern California, Los Angeles, California; \\ ${ }^{5}$ Department of Psychiatry, Keck School of Medicine, University of Southern California, Los Angeles, \\ California; and ${ }^{6}$ Division of Bioinformatics, Department of Preventive Medicine, Keck School of Medicine, \\ University of Southern California, Los Angeles, California
}

Object. Meningiomas are among the most common primary adult brain tumors. Although typically benign, roughly $2 \%-5 \%$ display malignant pathological features. The key molecular pathways involved in malignant transformation remain to be determined.

Methods. Illumina expression microarrays were used to assess gene expression levels, and Illumina singlenucleotide polymorphism arrays were used to identify copy number variants in benign, atypical, and malignant meningiomas (19 tumors, including 4 malignant ones). The authors also reanalyzed 2 expression data sets generated on Affymetrix microarrays $(n=68$, including 6 malignant ones; $n=56$, including 3 malignant ones). A weighted gene coexpression network approach was used to identify coexpression modules associated with malignancy.

Results. At the genomic level, malignant meningiomas had more chromosomal losses than atypical and benign meningiomas, with average length of 528, 203, and 34 megabases, respectively. Monosomic loss of chromosome 22 was confirmed to be one of the primary chromosomal level abnormalities in all subtypes of meningiomas. At the transcriptome level, the authors identified 23 coexpression modules from the weighted gene coexpression network. Gene functional enrichment analysis highlighted a module with 356 genes that was highly related to tumorigenesis. Four intramodular hubs within the module $(G A B 2, K L F 2, I D 1$, and $C T F 1)$ were oncogenic in other cancers such as leukemia. A putative meningioma tumor suppressor $M N 1$ was also identified in this module with differential expression between malignant and benign meningiomas.

Conclusions. The authors' genomic and transcriptome analysis of meningiomas provides novel insights into the molecular pathways involved in malignant transformation of meningiomas, with implications for molecular heterogeneity of the disease.

(http://thejns.org/doi/abs/10.3171/2013.10.FOCUS13326)

\section{KEY WORDS • meningioma • copy number alteration • WGCNA • hub genes}

$\mathrm{M}$ ENINGIOMAS are common intracranial tumors that arise from the meninges, the membranous covering of the brain and spinal cord. They constitute $25 \%-30 \%$ of all primary brain tumors, with an annual incidence of 4-6 per 100,000 persons. ${ }^{43,53}$ Most meningiomas are considered benign and grow slowly. ${ }^{51}$ The WHO classifies meningiomas into 3 subtypes: Grade I (benign meningioma), Grade II (atypical meningioma), and Grade III (malignant/anaplastic meningioma). The

\footnotetext{
Abbreviations used in this paper: SNP = single-nucleotide polymorphism; UCLA = University of California, Los Angeles; WGCNA = weighted gene correlation network analysis.
}

prevalence rates of these subtypes are roughly $80 \%-90 \%$, $6 \%-15 \%$, and $2 \%-5 \%$, respectively, based on several reports. ${ }^{28,35}$ Malignant meningiomas are typically characterized by aggressive behavior, with infiltration into the adjacent brain, focal necrosis, and increased vascularity.

\section{Disclaimer}

This article provides links to supplemental material on an external website hosted on the file server in Dr. Kai Wang's lab at the University of Southern California. These links are provided for informational purposes and as a convenience to the reader. Provision of these links does not imply any endorsement or approval of the website or its contents by the JNS Publishing Group or the American Association of Neurosurgeons. 


\section{Chang et al.}

Atypical and malignant meningiomas are often associated with high recurrence rates, even following complete resection, and are associated with shorter progressionfree and overall survival compared with their benign counterparts. ${ }^{43}$ Currently, the mechanisms underlying malignant transformation in meningiomas are poorly understood. Although several genetic studies have been performed on meningiomas to identify disease-susceptibility genes, few have focused on genetic and chromosomal alterations that may specifically contribute to the malignant transformation of these tumors.

Prior investigations have demonstrated an association between monosomic loss of chromosome 22 and meningioma formation. ${ }^{40}$ Other chromosome losses have been reported on 1p, 3q, 6q, 9p, 10q, 14q, 17p, 18p, 18q, and $22 \mathrm{q} .{ }^{22}$ Relative levels of chromosomal abnormalities have been noted to correlate with higher meningioma grade. ${ }^{23}$ A recent study indicated that benign meningiomas with alterations in chromosome 14 may have a predilection for aggressive behavior and recurrence. ${ }^{36} \mathrm{~A}$ previous genome-wide association study revealed that a common variant at 10p12.31 near MLLT10 influences the risk of meningioma. This was the first genome-wide association study signal reporting on this disease and expanded our knowledge of genetic events to that initiate tumorigenesis. ${ }^{8}$ Studies on the NF2 gene in sporadic meningiomas suggest that approximately one-third to one-half of these tumors have an inactivating mutation, often accompanied by loss of the other allele. ${ }^{40}$ Other candidate tumor suppressor genes that have been suggested to promote meningioma formation include $D A L-1, B A M 22, M N 1$, and $L A R G E .^{43}$ However, current knowledge is insufficient to fully understand the genetic underpinnings of this disease.

Genome-wide expression profiling analysis has also identified differentially expressed genes and pathways in meningiomas. ${ }^{10,18,23}$ Several tumor suppressor genes are downregulated in malignant meningiomas, compared with benign and atypical ones. ${ }^{10}$ Furthermore, a specific deregulated pathway with enrichment of underexpressed genes regulated by the transcription factors $S p l$ and $A G P /$ $E B P$ was identified in fibroblastic meningiomas compared with normal dura. ${ }^{18} \mathrm{~A}$ recent study suggested that meningiomas can be classified into 5 groups based on gene expression profiles. ${ }^{23}$

In the current study, we collected a sample set of 19 resected meningiomas comprising all 3 WHO grades, and we performed DNA copy number analysis using single-nucleotide polymorphism (SNP) genotyping arrays. In parallel, we also performed transcriptome analysis using gene expression microarrays to complement the copy number analysis. Considering the relatively small sample size, we also reanalyzed 2 publicly available meningioma expression data sets from the GEO database, totaling 9 additional malignant samples. Finally, we take advantage of weighted gene coexpression network analysis to identify dense coexpression subnetworks in meningiomas and detect oncogenic modules that are associated with malignant meningiomas. Our integrative genomics analysis yielded novel insights into the malignant transformation of meningiomas.

\section{Methods}

\section{Gene Expression Microarray}

The study was approved by the University of Southern California institutional review board. Written informed consent was obtained from all patients prior to collection of surgical tumor specimens. Following selection of benign, atypical, and malignant meningioma case samples with confirmed histopathology, total RNA samples were extracted from fresh-frozen meningioma tissue following a Qiagen RNA extraction protocol. A liquid nitrogen grinding method was used to prepare tissue samples for extraction. First, tissue samples were removed from the $-80^{\circ} \mathrm{C}$ refrigerator, cut into fragments, and weighed immediately to ensure that no more than $100 \mathrm{mg}$ tissue was present in every $1.5-\mathrm{ml}$ tube. Next, liquid nitrogen was added into the tube as soon as possible, followed by tissue grinding. Next, $900 \mu \mathrm{l}$ of QIAzol Lysis Reagent is added to each tube, vortexed for at least 60 seconds, and stored at room temperature $\left(15^{\circ} \mathrm{C}-25^{\circ} \mathrm{C}\right)$ for 5 minutes. The standard protocol of the RNeasy Kit is then applied to each tissue sample, with the entire process being performed on ice (Rneasy Plus Universal Mini Kit, Cat. no. 73404). The quality of total RNA was assessed using an Experion RNA StdSens Chip on a Bio-Rad bioanalyzer. Genome-wide expression profiles of approximately 47,000 transcripts were quantified using an Illumina $\mathrm{Hu}-$ manHT-12 v4 Expression BeadChip. Raw data were processed and normalized without background subtraction using the Illumina GenomeStudio software suite. Among the 19 samples, mRNA from 11 samples (5 benign, 2 atypical, and 4 malignant meningiomas) were successfully extracted with an RNA integrity number value over 7. Considering the small sample size, we added 1 more malignant sample to perform the DNA SNP microarray assay.

\section{Single-Nucleotide Polymorphism Array}

DNA extraction was performed from 10 to $20 \mathrm{mg}$ of tumor fragments using the DNeasy Blood \& Tissue kit from (Qiagen), according to the supplied protocol. Genomic DNA (250 ng) was assayed by the HumanOmniExpress BeadChip SNP array (Illumina) with 730,525 markers, including 392,197 SNP markers. All microarray analysis was performed at the USC Epigenome Center. Raw microarray data were processed by the GenomeStudio Software (Illumina) to generate $\log \mathrm{R}$ ratio and $\mathrm{B}$ allele frequency values for each marker on the array.

\section{Data Analysis}

Copy number alternations in 19 meningioma genomes were detected with a computational tool called OncoSNP from the SNP genotyping data. ${ }^{56}$ Both stromal contamination and intratumor heterogeneity models were activated in OncoSNP to perform a joint analysis and estimate the baseline level of stromal contamination and intratumor heterogeneity. This is the most accurate analysis at the expense of computing resources. The SNP genotyping arrays show "genomic wave patterns" in which signal intensity is correlated to local guanine-cytosine content, 
so the signal intensity value was adjusted by the genomic_wave.pl program in the PennCNV package. ${ }^{7,50}$ We used an $\mathrm{R}$ package LIMMA in Bioconductor to performed pairwise class distinction to identify genes differentially expressed among the benign, atypical, and malignant meningiomas (false discovery rate adjusted p value $<0.01$ ). ${ }^{47}$ Since no differentially expressed genes were found in atypical and benign samples, we reanalyzed the microarray data by combining atypical and benign samples and comparing these to malignant samples. Two hundred eighty-eight differentially expressed genes were finally identified between malignant and benign-atypical samples. Copy number alternations and gene expression data were clustered using MultiExperiment Viewer software suite v.4.7. ${ }^{29}$ We used DAVID web server (http://david. abcc.ncifcrf.gov/) to test enrichment in gene sets with GO, SwissProt, and InterPro terms compared with the background list of all genes. ${ }^{15}$

We also downloaded raw data (as CEL files) for 2 meningioma whole-genome gene expression studies from the GEO database (accession nos. GSE16581 and GSE4780, respectively). These samples were assayed on the Affymetrix Human Genome U133 Plus 2.0 platform, so we used the Affymetrix Expression Console software to preprocess the CEL files with Robust MultiChip Analysis protocol and then exported the probeset-level expression levels as a tab-delimited text file. The LIMMA package in $\mathrm{R}^{47}$ was used to identify differentially expressed genes.

\section{Weighted Gene Coexpression Network Analysis}

To construct a network from gene expression data, we selected 3600 of the most varying genes and calculated the Pearson correlation for all pairs of selected genes. The correlation matrix was converted into an adjacency matrix with a power function, so that the connection strength between 2 genes $x_{i}$ and $x_{j}$ was defined as $a_{i j}=\left|\operatorname{cor}\left(x_{i}, x_{j}\right)\right|^{\beta}$. The parameter $\beta$ was determined by the criterion that the resulting adjacency matrix approximately fit a scale-free topological feature according to a previously proposed model-fitting index. ${ }^{57}$ The model-fitting index of a perfect scale-free network is 1 . Here, we chose the smallest value of $\beta(\beta=9)$, which is the minimum value required to make the model-fitting index 0.85 (Fig. 1). ${ }^{9,57}$

The adjacency matrix was further transformed into a topological overlap matrix, which captures not only the direct interaction between 2 genes but also their indirect interactions through all the other genes in the network. A similarity measure was defined:

$$
T O M_{i j}=\frac{\sum_{u} a_{i u} a_{u j}+a_{i j}}{\min \left(k_{i}, k_{j}\right)+1-a_{i j}},
$$

where

$$
k_{i}=\sum_{u} a_{i u}
$$

is the node connectivity. ${ }^{42,57}$ Subsequently, $1-$ TOM $_{i j}$ was used as a distance matrix in the hierarchical clustering of the transcript units for module detection. ${ }^{42}$

\section{Oncogenic Module Analysis}

In the oncogenic module, member genes pairs with a Mutual Rank (http://coxpresdb.jp/help/mr.shtml) < 200 or Pearson correlation coefficient $>0.4$ are considered connected..$^{33}$ The core region was identified with MCODE, a plugin planted in Cytoscape. ${ }^{1,44}$ Protein-protein interaction information was queried from Michigan Molecular Interactions (MiMI). ${ }^{17}$ The network topology was visualized with Cytoscape. ${ }^{44}$

\section{Results}

\section{Chromosomal Profiles of Genetic Alternations}

We assayed copy number alteration profiles in 19 histopathologically verified human meningioma tumor
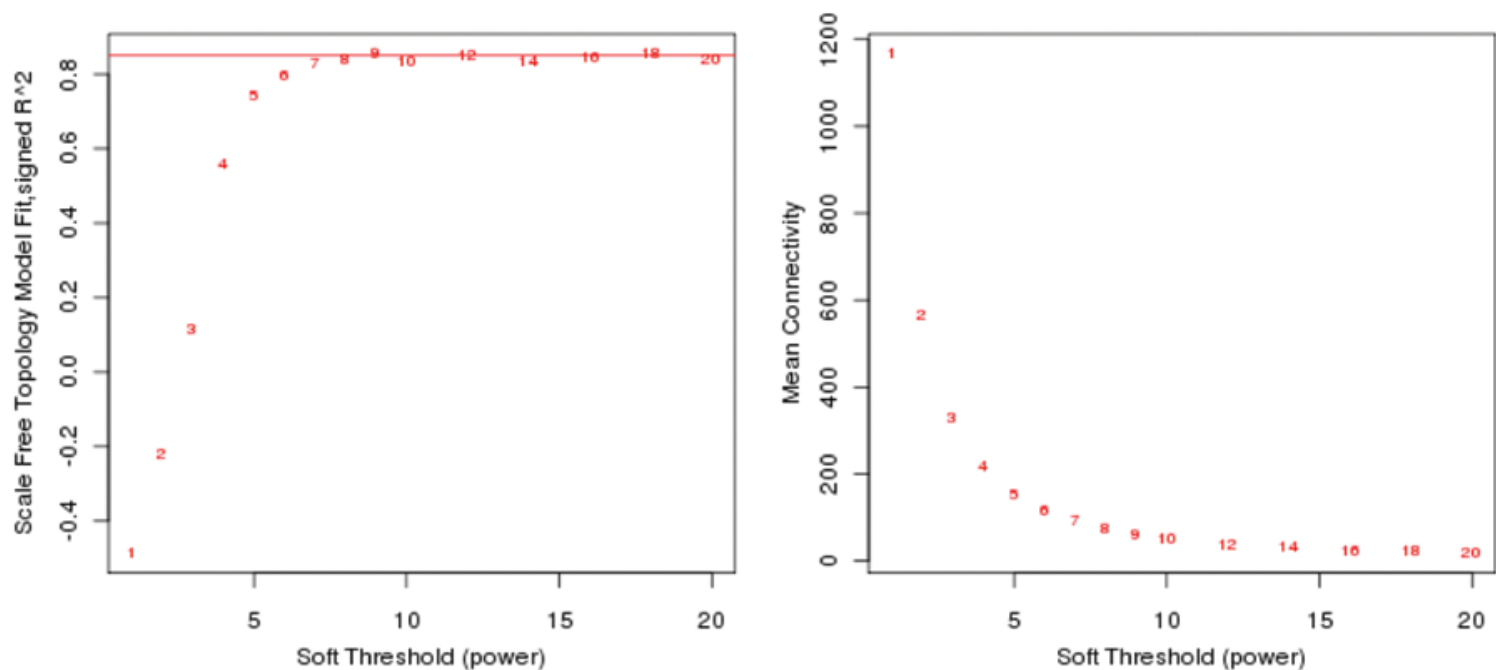

FiG. 1. Analysis of network topology for various soft-thresholding powers. Left: The scale-free fit index (y axis) as a function of the soft-thresholding power (x axis). Right: The mean connectivity (in degree, $y$ axis) as a function of the soft-thresholding power (x axis). 
samples: 10 benign (WHO Grade I), 5 atypical (WHO Grade II), and 4 malignant (WHO Grade III) meningiomas (Fig. 2). The average length of copy number gains was 423,701 , and 270 megabases in benign, atypical, and malignant meningiomas, respectively. In comparison, the average length of copy number loss was 34, 203, and 528 megabases in benign, atypical, and malignant meningiomas, respectively. In general, hierarchical clustering analysis classified the samples into 2 groups. Three malignant samples and 1 atypical sample with significant gain or loss of chromosomes were clustered into a small group. One malignant, 2 atypical, and all 10 benign samples with fewer chromosomal abnormalities were clustered into a separate larger group. The larger group could further be divided into 2 additional subgroups based on the relative degree of copy number amplification. The chromosomal copy number patterns suggested that higher grades of meningiomas demonstrate more variability in their genomic structures, especially with regard to genomic losses.

\section{High Chromosomal Arm-Level Abnormality Occurrences in High-Grade Meningiomas}

Previous studies have suggested that a strong association between increased frequency of chromosomal losses with increased histological grades exists in meningiomas. ${ }^{34}$ Our copy number variation analysis corroborated the findings that malignant and atypical meningiomas present more chromosomal arm-level abnormalities than benign meningiomas (Fig. 2). Obvious deletion or amplification events in chromosomal arms were found in 2 of 4 malignant samples (Sample ID 249: amplification on 3 chromosomal arms, deletion on 9 chromosomal arms; Sample ID 255: deletion on 3 chromosomal arms) and 2 of 4 atypical samples (Sample ID 351: amplification on 17 chromosomal arms; Sample ID 678: amplification on 4 chromosomal arms, deletion on 6 chromosomal arms), whereas only 1 arm-level deletion occurred in 1 of 15 benign samples. Three of 4 malignant samples showed a monosomic loss of chromosome 4, but this event was not observed in atypical and benign samples. However, we also found a malignant sample (Sample ID 254) and an atypical sample (Sample ID 489) that showed a significant repression of chromosomal alternations compared with other samples from the same WHO grade (Fig. 2). Thus, other mechanisms may be involved in the development and malignant transformation of meningiomas.

\section{Loss of Chromosome 22}

Our results also showed that nearly half of meningioma samples have significant deletions in chromosome 22q, including 2 of 4 malignant samples, 2 of 4 atypical samples, and 5 of 15 benign samples (Fig. 2). To confirm this observation, we applied a statistical framework to distinguish genuine chromosomal aberrations from random background events. ${ }^{4}$ The results showed that loss of chromosome $22 \mathrm{q}$ was clearly statistically significant $(\mathrm{Q}$ value $=0$; Table 1). In addition, loss of chromosome $1 \mathrm{p}$ was also detected with high statistical significance $(\mathrm{Q}$ value $=0.0484$; Table 1$)$. Loss of chromosome 22q may be a primary event with respect to chromosomal abnormalities in meningiomas, as demonstrated by the few benign samples with monosomy of chromosome 22 and loss of chromosome 22 in most high-grade meningiomas. ${ }^{23}$

\section{Gene Expression Analysis}

Hierarchical clustering analysis indicated that 5 benign and atypical samples were represented by similar whole-genome expression patterns and were grouped closely together. The malignant samples, however, showed varying patterns and were not clustered together. These observations imply that gene expression profiles of malignant meningiomas are likely to be disordered compared

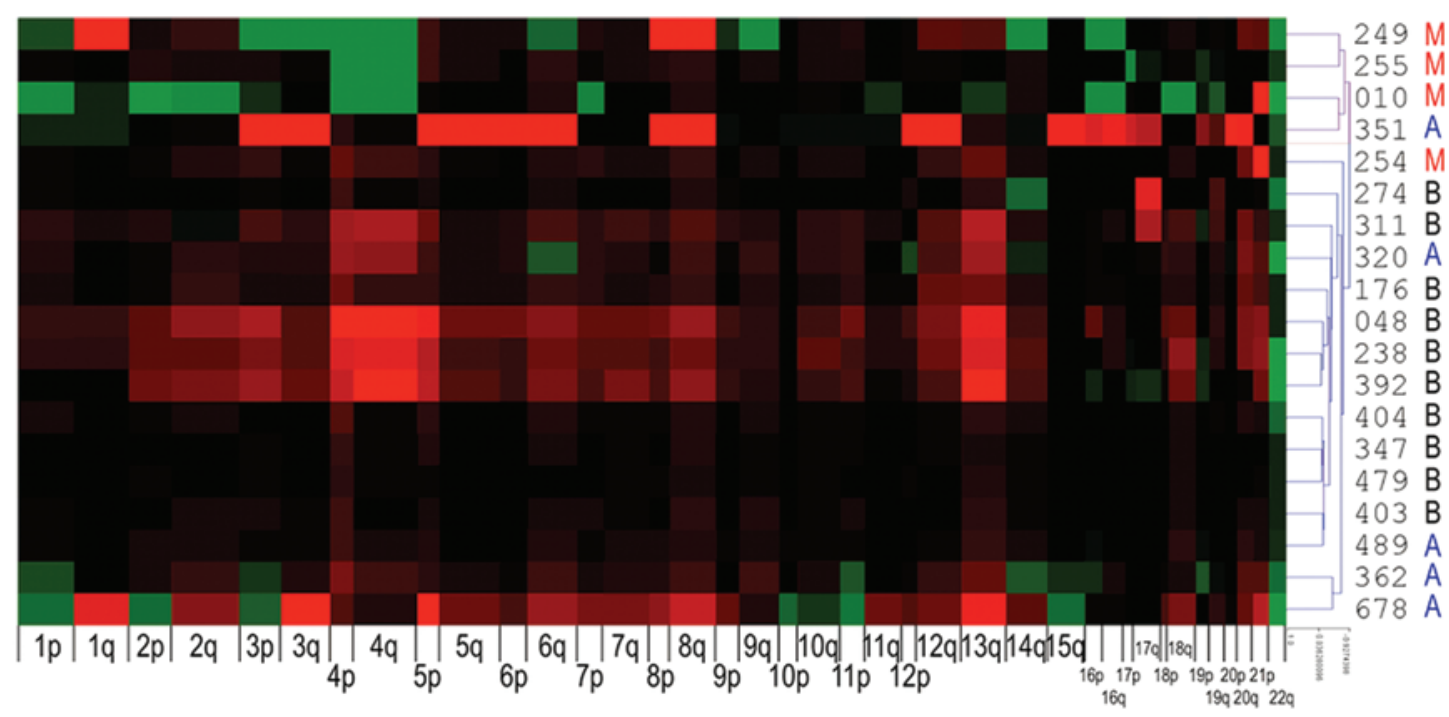

Fig. 2. Chromosomal profiles of genomic copy number alternations and hierarchical clustering results of 19 meningioma samples. The 2 major clusters are colored by red and blue. Copy numbers of the genomic regions are marked from green (loss), black (normal), and red (gains). The regions of 13p, 14p, 15p, 21p, and 22p are ignored here, since they are not well covered by the SNP array data. The malignant samples are separated from benign/atypical samples, except for one atypical sample (Sample ID 351). $A$ = atypical; $B$ = benign; $M=$ malignant. 
An oncogenic functional module in meningioma

TABLE 1: Significance of chromosomal arm abbreviations predicted by GISTIC software*

\begin{tabular}{|c|c|c|c|c|c|c|c|}
\hline \multirow[b]{2}{*}{ Arm } & \multirow[b]{2}{*}{ No. of Genes } & \multicolumn{3}{|c|}{ Amplification } & \multicolumn{3}{|c|}{ Deletion } \\
\hline & & Frequency & z-Score & Q Value & Frequency & z-Score & Q Value \\
\hline $1 p$ & 1423 & 0 & -1.17 & 0.887 & 0.26 & 2.68 & $0.0484 \dagger$ \\
\hline $1 q$ & 1274 & 0 & -1.21 & 0.887 & 0.16 & 1.16 & 0.433 \\
\hline $2 p$ & 590 & 0 & -1.03 & 0.887 & 0.16 & 1.74 & 0.202 \\
\hline $2 q$ & 1017 & 0 & -1.18 & 0.887 & 0.11 & 0.488 & 0.623 \\
\hline $3 p$ & 661 & 0.06 & -0.0907 & 0.887 & 0.11 & 0.812 & 0.508 \\
\hline $3 q$ & 732 & 0.11 & 0.76 & 0.887 & 0.06 & -0.127 & 0.77 \\
\hline $4 p$ & 291 & 0 & -0.94 & 0.887 & 0.16 & 2.07 & 0.152 \\
\hline $4 q$ & 667 & 0 & -1.05 & 0.887 & 0.16 & 1.66 & 0.21 \\
\hline $5 p$ & 169 & 0.05 & 0.0851 & 0.887 & 0 & -0.957 & 0.887 \\
\hline $5 q$ & 1009 & 0.05 & -0.376 & 0.887 & 0 & -1.21 & 0.887 \\
\hline $6 p$ & 768 & 0.06 & -0.204 & 0.887 & 0.06 & -0.204 & 0.77 \\
\hline $6 q$ & 550 & 0.06 & -0.0317 & 0.887 & 0.11 & 0.897 & 0.481 \\
\hline $7 p$ & 390 & 0 & -1.03 & 0.887 & 0.05 & -0.0551 & 0.77 \\
\hline $7 q$ & 815 & 0 & -1.16 & 0.887 & 0.05 & -0.284 & 0.77 \\
\hline $8 p$ & 370 & 0.11 & 0.964 & 0.887 & 0 & -0.994 & 0.887 \\
\hline $8 q$ & 554 & 0.11 & 0.811 & 0.887 & 0 & -1.05 & 0.887 \\
\hline $9 p$ & 275 & 0 & -1.02 & 0.887 & 0 & -1.02 & 0.887 \\
\hline $9 q$ & 731 & 0 & -1.13 & 0.887 & 0.05 & -0.242 & 0.77 \\
\hline $10 p$ & 248 & 0 & -0.956 & 0.887 & 0.11 & 1.08 & 0.458 \\
\hline $10 q$ & 817 & 0 & -1.12 & 0.887 & 0.11 & 0.619 & 0.615 \\
\hline $11 p$ & 578 & 0 & -1.02 & 0.887 & 0.16 & 1.75 & 0.202 \\
\hline $11 q$ & 1048 & 0 & -1.19 & 0.887 & 0.11 & 0.469 & 0.623 \\
\hline $12 p$ & 374 & 0.05 & -0.0455 & 0.887 & 0 & -1.02 & 0.887 \\
\hline $12 q$ & 943 & 0.05 & -0.345 & 0.887 & 0 & -1.19 & 0.887 \\
\hline $13 q$ & 400 & 0 & -1.06 & 0.887 & 0 & -1.06 & 0.887 \\
\hline $14 q$ & 874 & 0 & -1.03 & 0.887 & 0.26 & 3.26 & 0.0109 \\
\hline $15 q$ & 776 & 0.06 & -0.208 & 0.887 & 0.06 & -0.208 & 0.77 \\
\hline $16 p$ & 585 & 0.06 & 0.012 & 0.887 & 0.17 & 1.85 & 0.202 \\
\hline $16 q$ & 456 & 0.06 & 0.0209 & 0.887 & 0.11 & 0.973 & 0.46 \\
\hline $17 p$ & 420 & 0.06 & 0.0417 & 0.887 & 0.11 & 1 & 0.46 \\
\hline $17 q$ & 1086 & 0.06 & -0.293 & 0.887 & 0.11 & 0.527 & 0.623 \\
\hline $18 p$ & 93 & 0 & -0.932 & 0.887 & 0.05 & 0.138 & 0.755 \\
\hline $18 q$ & 265 & 0 & -0.989 & 0.887 & 0.05 & 0.022 & 0.77 \\
\hline $19 p$ & 721 & 0 & -1.03 & 0.887 & 0.21 & 2.53 & 0.0552 \\
\hline $19 q$ & 1055 & 0 & -1.15 & 0.887 & 0.16 & 1.33 & 0.36 \\
\hline $20 p$ & 249 & 0.11 & 1.16 & 0.887 & 0.06 & 0.147 & 0.755 \\
\hline $20 q$ & 528 & 0.11 & 0.914 & 0.887 & 0.06 & -0.0196 & 0.77 \\
\hline $21 q$ & 324 & 0.17 & 2.14 & 0.636 & 0.06 & 0.162 & 0.755 \\
\hline $22 q$ & 598 & 0 & -0.773 & 0.887 & 0.53 & 8.38 & $0 \dagger$ \\
\hline
\end{tabular}

* GISTIC = Genomic Identification of Significant Targets in Cancer.

$\dagger$ Values are below 0.05 , indicating that the copy number variations in this chromosome are significantly associated with disease. 
with benign and atypical tumors (Fig. 3). We identified 260 differentially expressed genes between malignant and benign samples and 59 differentially expressed genes between malignant and atypical samples. Interestingly, we did not identify any differentially expressed genes between benign and atypical samples (Fig. 4). In a previous study, we also demonstrated that genome-wide methylation patterns cannot differentiate benign and atypical meningiomas but can easily differentiate malignant from benign/atypical samples. ${ }^{11}$ Considering the small sample size for the atypical group and the low power to detect differential expression, we combined the benign and atypical samples and subsequently identified 288 differentially expressed genes between the malignant and benign/atypical sample groups. Hierarchical clustering analysis confirmed that the differentially expressed genes were clearly divided into these malignant and nonmalignant groups (Fig. 5).

Notably, a probable tumor suppressor gene, $M N 1$, showed significant repression in all the malignant samples analyzed in our study (Table S1, http://bionas.usc.edu/ meningioma/Table_S1.xlsx). Previous research also suggests that inactivation of the MN1 gene in meningiomas may contribute to their pathogenesis. ${ }^{24} \mathrm{We}$ also found that the IGFBP5 gene (whose promoter transcription can be activated by the $M N 1$ gene product) was significantly suppressed (Table S1). ${ }^{31}$ Interestingly, MN1 can both stimulate and inhibit transcription of this gene. Overexpression of $M N 1$ was also reported in the development of acute myeloid leukemia. ${ }^{30}$

Considering the relatively small sample size, to further validate our gene expression result, we downloaded the raw data from 2 meningioma gene expression studies from GEO, hereafter referred to as the University of California, Los Angeles (UCLA) study (GSE16581, 63 samples) ${ }^{23}$ and

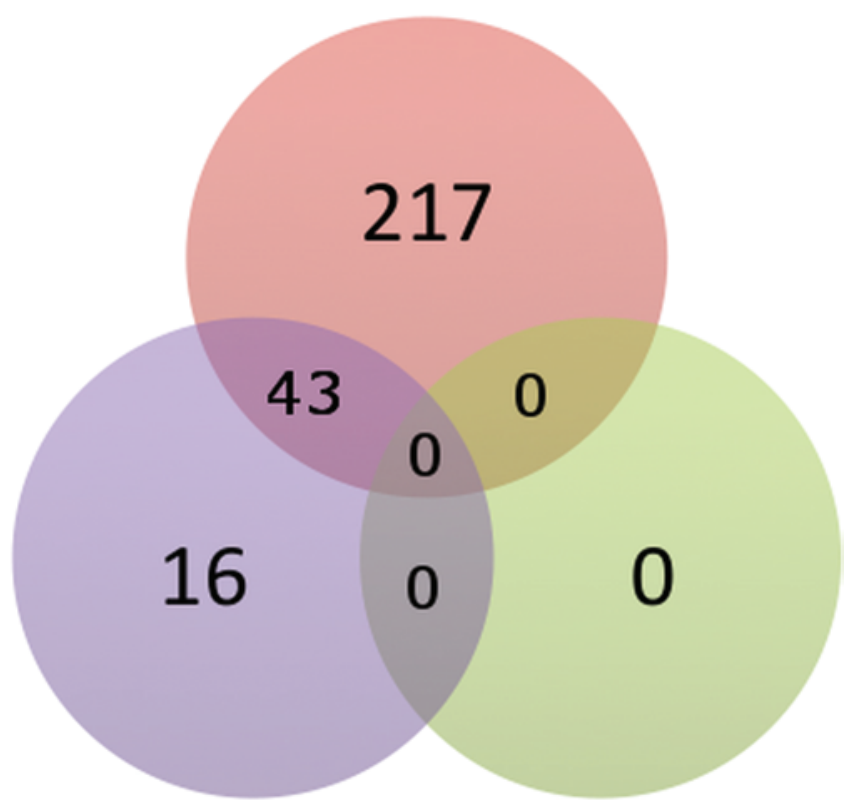

Fig. 4. Venn diagram of differentially expressed genes among 3 classifications of meningiomas. Red circle shows the number of differentially expressed genes between malignant and benign meningiomas, purple circle shows the number of differentially expressed genes between malignant and atypical meningiomas, and green circle shows the number of differentially expressed genes between benign and atypical meningiomas.

the Scheck study (GSE4780, 56 samples). We identified 410 and 465 genes differentially expressed between benign and malignant samples from the UCLA and Scheck study, respectively. However, the number of overlapping genes among the 3 data sets is low: only 3 common genes were identified: $L E P R, F X Y D 5$, and KCNMA1. LEPR was

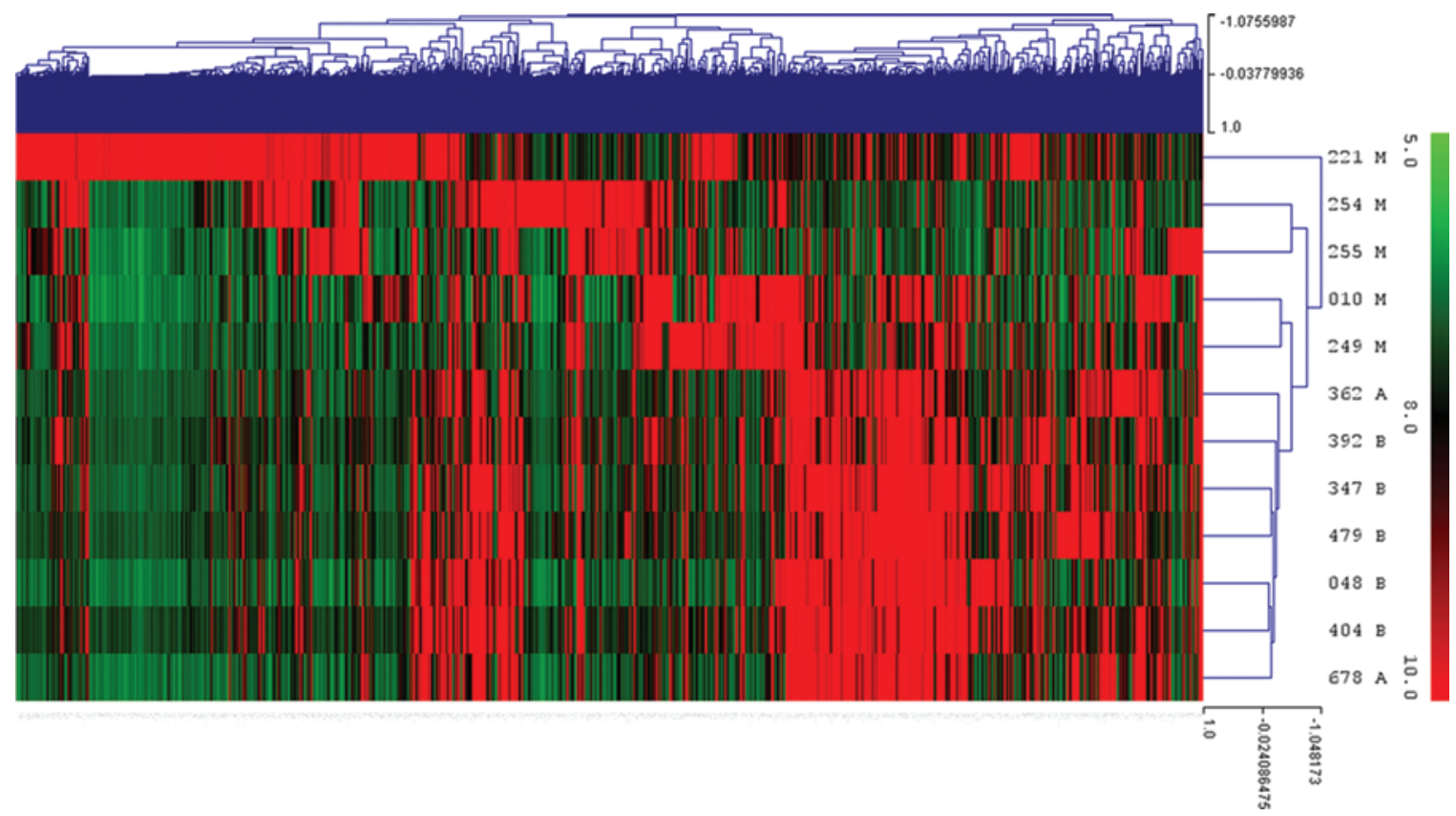

FıG. 3. Hierarchical clustering results of transcriptome data using the 1000 most variably expressed genes. Genes with high, medium, and low expression values are colored by red, black, and green, respectively. The malignant samples are clearly separated from benign/atypical samples. 


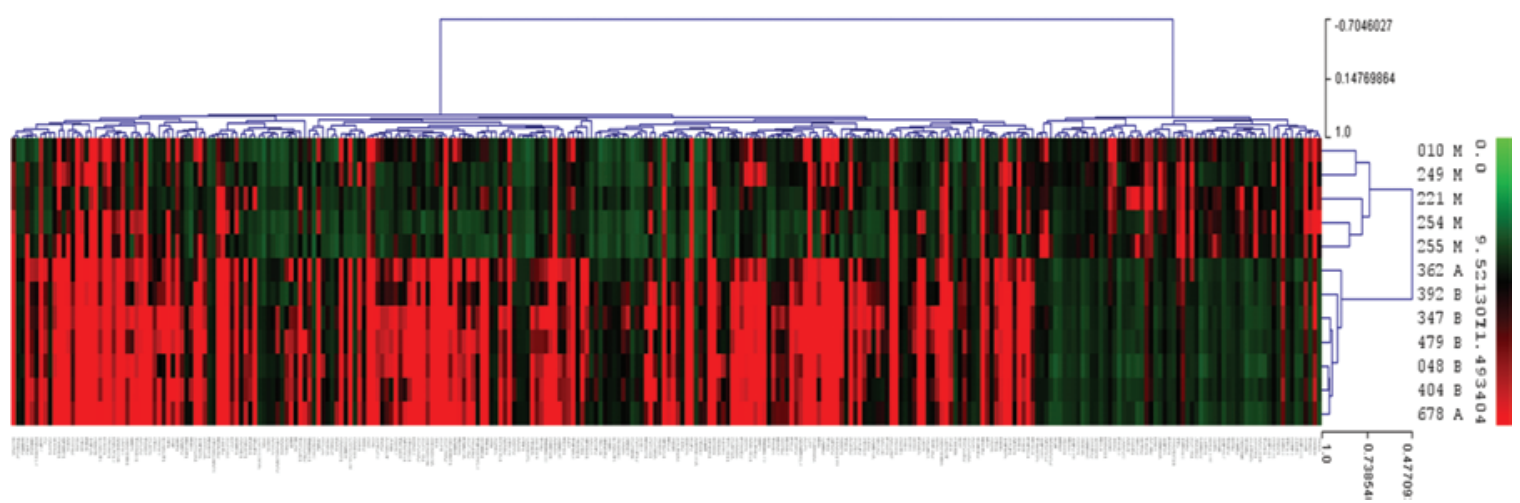

FIG. 5. Hierarchical clustering of 288 genes with differential expression between 5 malignant groups and 5 benign and 2 atypical groups. The cluster is color coded using red for upregulation, green for downregulation, and black for median expression.

associated with pituitary adenoma, ${ }^{12,20,46}$ FXYD5 was associated with a broad spectrum of cancers, and KCNMAI was also associated with giloma., 3,27,41 The number of overlapping genes between each pair of the 3 data sets was higher: 26 between the UCLA and USC studies, 34 between the Scheck and USC studies, and 35 between the UCLA and Scheck studies. Interestingly, we found that the MN1 gene was also differentially expressed in the UCLA data set. We next mapped the identified differentially expressed genes from the 3 data sets on the Cancer Genome Anatomy Project by DAVID and extracted the top 10 significantly enriched pathways (Table S2, http://bionas.usc. edu/meningioma/Table_S2.xlsx). For our data set, the top 4 pathways were all relevant to meningiomas, indicating a strong correlation of our data set and the Cancer Genome Anatomy Project. The first and third most significantly enriched pathways in the Scheck study were also related to meningiomas. However, no pathway associated with meningiomas was detected in the UCLA study.

\section{Association Between DNA Copy Number and Gene} Expression Levels

We calculated the Pearson correlation coefficient between DNA copy number and gene expression level. Our results showed that a certain proportion of genes were closely correlated (Pearson correlation coefficient $>0.5$; Fig. 6). Gene ontology enrichment analysis suggested that genes related to mitochondrial function were significantly enriched in DNA copy number and correlation to high gene expression (Table S3, http://bionas.usc.edu/meningioma/ Table_S3.xlsx). Analysis using Fisher's exact test also lent support to the hypothesis that these genes are enriched in chromosome regions with clear copy number abnormalities (chromosome 22q13.1 [p = 2.6e-5], chromosome 4p16.3 [p = 1.15e-8]; Table S3).

Previous studies on meningiomas revealed several aberrant signaling pathways that may be involved in tumorigenesis. ${ }^{39}$ These pathways include membrane-associated protein 4.1 family-relevant pathways, vascular endothelial growth factor-involved angiogenic pathways, Hedgehog signaling pathway, MAPK and PI3K signaling pathways, Notch signaling pathway, and growth factorand cytokine-induced pathways. We next examined our copy number variation and gene expression data with respect to the candidate meningioma pathways. We found that copy number alterations disrupt genes in some of these pathways and may result in gene expression changes. Besides MN1, a few genes in the PI3K/Akt pathway and some bone morphogenetic proteins were also differentially expressed between the benign/atypical and malignant samples. These results suggested that these pathways may play a role in tumor progression toward malignancy (Table S4, http://bionas.usc.edu/meningioma/Table_S4.xlsx).

\section{An Oncogenic Module Identified by Weighted Gene Coexpression Networks}

We further analyzed the gene expression data with the weighted gene correlation network analysis (WGCNA) algorithm, which has been widely applied in gene coexpression network construction and module detections. ${ }^{5,16,21,32,57}$ Twenty-three coexpression modules were identified (Fig. 7). Functional annotations of these modules were performed by analyzing the gene compositions within each module. Three modules were significantly enriched in genes with specific functional categories-Module I: glycoprotein/membrane/blood vessel development/cell migration, 356 genes; Module II: cell cycle, 97 genes; and Module III: synapse/membrane, 1289 genes. Of these, Module I is considered an oncogenic module since it is enriched in cancer-related functional processes. Not surprisingly, the differentially expressed gene $M N 1$, as well as its target gene IGFBP5, was also contained within this oncogenic module.

In a biological network, a few highly connected

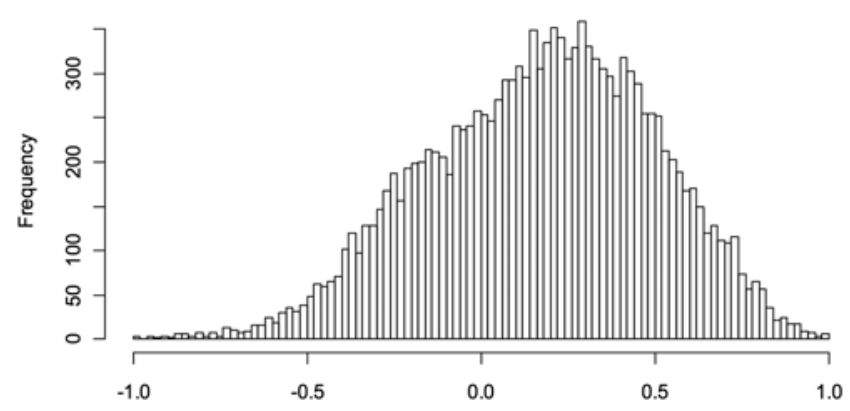

FIG. 6. Distribution of DNA number and expression correlation. The $x$ axis represents the Pearson correlation coefficient, and the $y$ axis represents the frequency. The average is significantly higher than zero $(p=0)$. 


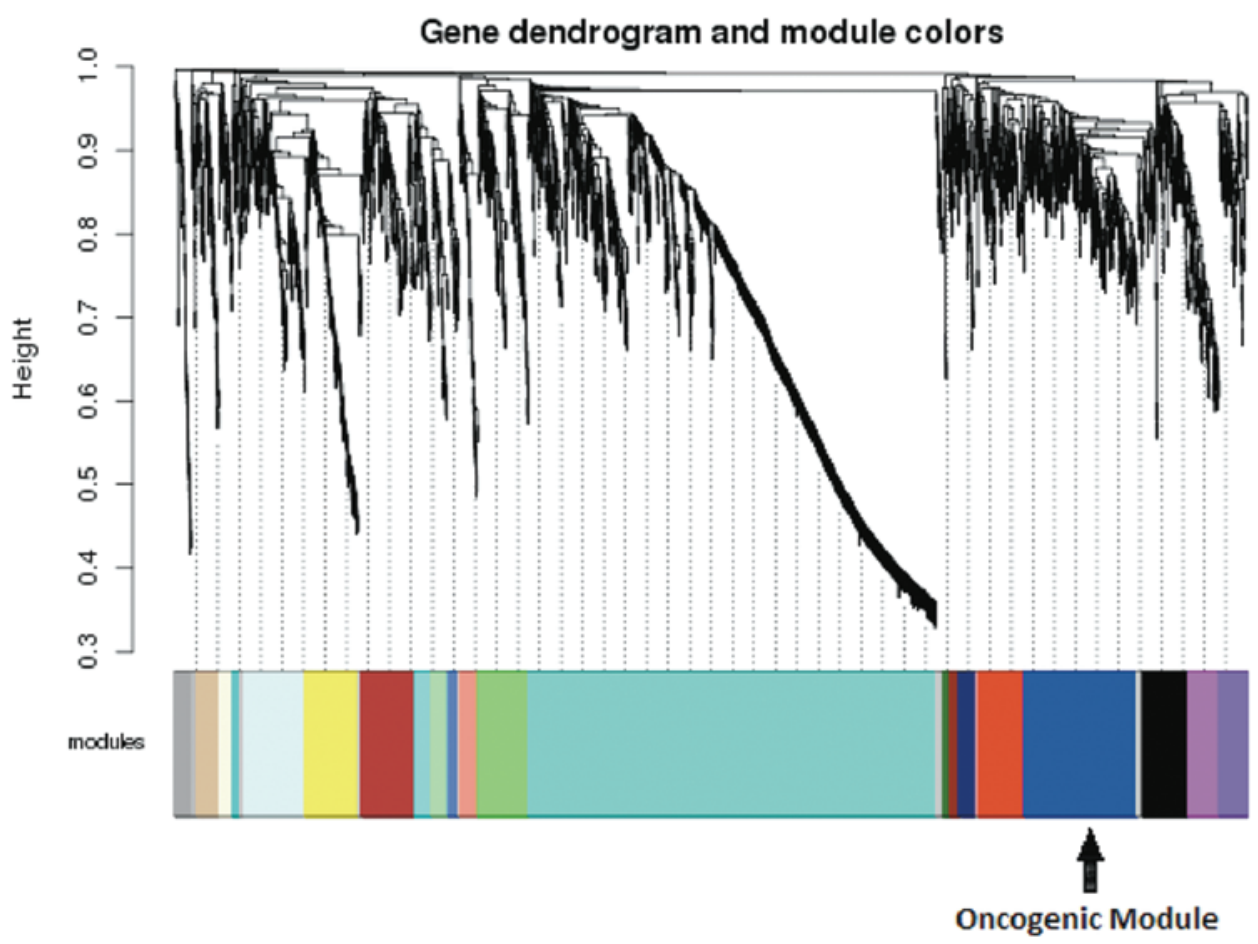

FIG. 7. Weighted gene coexpression network identified multiple functional modules. The upper portion of the figure is the cluster dendrogram of genes, and the lower section shows the modules of coexpressed genes. Genes within the same module are colored in the same color.

nodes that hold the whole network together are referred to as hubs ${ }^{48}$ Similarly, genes with a high number of interactions are believed to play an important role in organizing the biological process of functional modules. ${ }^{2,13,48} \mathrm{We}$ therefore zoomed in to identify hub genes in the oncogenic module. Unlike classic gene coexpression networks or protein-protein interaction networks, WGCNA defined a whole network connectivity measure $\left(\mathrm{k}_{\text {Total }}\right)$ for each gene, based on its Pearson correlation coefficient with all of the other genes, and an intramodular connectivity measure $\left(\mathrm{k}_{\mathrm{w} \text { ithin }}\right)$ when only considering the connection strength of each gene with all the other genes within the specific module. ${ }^{57}$ In this study, the intramodular connectivity is far more meaningful than whole network connectivity in the oncogenic module. We hypothesized that intramodular hub genes may be associated with meningioma tumorigenesis. As expected, 2 intramodular hub genes (GAB2 and KLF2) with the highest values of connectivity were related to tumorigenesis, and 2 additional intramodular hub genes (IDI and CTFI) are known oncogenes (Table 2). These 4 hub genes were also significantly differentially expressed between malignant and benign meningiomas (Table S1). Interestingly, the 4 hub genes are also related to leukemia. ${ }^{25,37,52,54}$ As described above, the meningioma tumor suppressor $M N 1$ in this module was also associated with leukemia. ${ }^{26,30}$

\section{Validation of the Oncogenic Module With Other Biological Networks}

We further validated the oncogenic module with a coexpressed gene database, COXPRESdb, which collected large mammalian coexpression information from publicly available GeneChip data. ${ }^{33} \mathrm{We}$ created a subnetwork by mapping the genes of the oncogenic module onto the COXPRESdb database (Fig. 8). To further explore the oncogenic module in detail, we identified a highly connected core region of 27 genes with MCODE from the oncogenic module (Fig. 9; see Methods). The 27 core genes containing $G A B 2$ and $K L F 2$ are mostly related to cancer. $I D I$ and $C T F I$ are also directly connected with members of the core region (Fig. 7). Although MNI is not directly connected to the core region, the proteinprotein interaction networks show that $M N 1$ can connect to $K L F 2$ through EP300. These results show a layered structure in which $G A B 2$ and $K L F 2$ function as central hubs. Genes or gene products interacting with them can form a core region in the oncogenic module. Most of the genes in the module can associate with GAB2 and KLF2 through those in the core region.

\section{Discussion}

In this study, we compared different subtypes of meningiomas at both the genomic and transcriptome levels. Our results identified chromosomal level alternations and differentially expressed genes between benign and malignant meningiomas. Gene coexpression network analysis revealed a module enriched with oncogenes that may represent a potential developmental pathway in meningioma pathogenesis and malignant transformation.

At the genomic level, copy number alteration analysis revealed that the degree of chromosomal abnormality correlates with the WHO meningioma grade. Monosomic loss of chromosome 22 may be considered a significant 
An oncogenic functional module in meningioma

TABLE 2: Connectivity strength of genes in the oncogenic module*

\begin{tabular}{|c|c|c|c|c|}
\hline Gene & $\mathrm{k}_{\text {Total }}$ & $\mathrm{k}_{\text {Within }}$ & $\mathrm{k}_{\text {Out }}$ & $k_{\text {Diff }}$ \\
\hline GAB2† & 91.937226 & 61.3776024 & 30.559624 & 30.8179788 \\
\hline$K L F 2 \dagger$ & 88.975097 & 61.065278 & 27.909819 & 33.15545911 \\
\hline SNORA59A & 93.758325 & 60.5207121 & 33.237613 & 27.28309894 \\
\hline PTGDR & 87.801063 & 59.7878167 & 28.013246 & 31.77457035 \\
\hline SNORA59B & 89.138849 & 57.4173046 & 31.721544 & 25.69576019 \\
\hline SLC47A1 & 93.172951 & 57.3408979 & 35.832053 & 21.50884502 \\
\hline SCARA5 & 92.73639 & 57.2757312 & 35.460659 & 21.81507259 \\
\hline CLIC2 & 84.49493 & 57.0510458 & 27.443885 & 29.60716128 \\
\hline PDZRN3 & 79.200217 & 55.7714971 & 23.42872 & 32.34277751 \\
\hline ENPP6 & 81.056553 & 53.4198296 & 27.636723 & 25.78310628 \\
\hline FGL2 & 80.553559 & 53.1927761 & 27.360783 & 25.83199294 \\
\hline ID1† & 93.686755 & 52.1946409 & 41.492114 & 10.70252651 \\
\hline THSD4 & 84.486848 & 51.3173816 & 33.169466 & 18.14791539 \\
\hline PDE9A & 77.034959 & 50.9068069 & 26.128152 & 24.77865462 \\
\hline CTF1† & 79.880531 & 50.8034799 & 29.077051 & 21.72642927 \\
\hline SULF2 & 81.416589 & 50.291748 & 31.124841 & 19.16690698 \\
\hline SLC26A2 & 79.16335 & 50.0251871 & 29.138163 & 20.88702448 \\
\hline KCNMA1 & 81.470279 & 50.0093559 & 31.460924 & 18.54843236 \\
\hline LRRN4CL & 75.794449 & 49.2979355 & 26.496514 & 22.8014219 \\
\hline LOC100131277 & 70.845041 & 48.6058186 & 22.239222 & 26.36659624 \\
\hline HSPB2 & 79.865166 & 48.3246837 & 31.540482 & 16.78420127 \\
\hline$E P H X 1$ & 79.476038 & 47.937519 & 31.538519 & 16.399 \\
\hline ST6GALNAC2 & 67.865026 & 47.9022072 & 19.962819 & 27.93938834 \\
\hline CTXN3 & 68.096972 & 47.4314341 & 20.665538 & 26.76589649 \\
\hline NHEDC2 & 71.413542 & 47.234591 & 24.178951 & 23.05563971 \\
\hline WNK4 & 72.822522 & 46.761346 & 26.061176 & 20.70016994 \\
\hline ST6GAL1 & 72.57842 & 46.6197313 & 25.958689 & 20.66104219 \\
\hline TMEM100 & 72.370632 & 46.0702591 & 26.300373 & 19.76988595 \\
\hline PGR & 69.890521 & 45.9499607 & 23.940561 & 22.00940005 \\
\hline SEMA6C & 72.651289 & 45.7996648 & 26.851624 & 18.94804063 \\
\hline SLC7A2 & 88.093278 & 45.5681683 & 42.52511 & 3.043058586 \\
\hline PENK & 60.904288 & 45.1546739 & 15.749614 & 29.40506027 \\
\hline DHRS3 & 71.615595 & 45.0212782 & 26.594317 & 18.426961 \\
\hline PGM5 & 66.511249 & 44.4179815 & 22.093267 & 22.32471413 \\
\hline SLPI & 83.390389 & 43.4470172 & 39.943371 & 3.503645876 \\
\hline TMOD1 & 72.801692 & 42.8815547 & 29.920138 & 12.96141702 \\
\hline SMAD9 & 61.961535 & 42.6894245 & 19.272111 & 23.41731393 \\
\hline MSRB3 & 73.598286 & 42.3308705 & 31.267416 & 11.06345497 \\
\hline PHGDH & 74.777432 & 42.2953639 & 32.482069 & 9.81329536 \\
\hline MAL2 & 58.90444 & 42.051725 & 16.852715 & 25.19901013 \\
\hline IGFBP5 & 64.650266 & 41.8840948 & 22.766171 & 19.11792343 \\
\hline PLEKHA6 & 75.35317 & 41.6674635 & 33.685706 & 7.981757175 \\
\hline C9ORF125 & 75.376087 & 40.8383805 & 34.537707 & 6.300673862 \\
\hline PODN & 63.918771 & 40.6111519 & 23.307619 & 17.30353258 \\
\hline PLCD4 & 70.390593 & 40.603878 & 29.786715 & 10.81716307 \\
\hline AFAP1L1 & 68.033303 & 40.441747 & 27.591556 & 12.8501905 \\
\hline LOC646347 & 61.70555 & 40.1098155 & 21.595734 & 18.514081 \\
\hline SLC7A1 & 59.581774 & 39.9054968 & 19.676277 & 20.22921977 \\
\hline HS.569162 & 52.549036 & 39.8293081 & 12.719727 & 27.10958066 \\
\hline
\end{tabular}

(continued) 
X. Chang et al.

TABLE 2: Connectivity strength of genes in the oncogenic module* (continued)

\begin{tabular}{|c|c|c|c|c|}
\hline Gene & $\mathrm{k}_{\text {Total }}$ & $k_{\text {Within }}$ & $\mathrm{k}_{\text {Out }}$ & $k_{\text {Diff }}$ \\
\hline SLC38A2 & 79.247877 & 39.5546778 & 39.693199 & -0.138521551 \\
\hline$A L P L$ & 69.246473 & 39.4975812 & 29.748892 & 9.748689668 \\
\hline LPAR5 & 61.829622 & 39.2725587 & 22.557063 & 16.71549586 \\
\hline$A D R B 2$ & 67.238236 & 39.2556038 & 27.982632 & 11.27297214 \\
\hline A4GALT & 78.239733 & 39.0776285 & 39.162105 & -0.084476175 \\
\hline C10ORF116 & 61.504405 & 38.5604319 & 22.943973 & 15.61645855 \\
\hline SOD3 & 55.24492 & 38.5499784 & 16.694941 & 21.85503705 \\
\hline LOC644348 & 53.888645 & 38.2882185 & 15.600427 & 22.68779164 \\
\hline LOC728715 & 65.672533 & 38.23127 & 27.441263 & 10.7900071 \\
\hline LOC642590 & 58.959018 & 38.1160477 & 20.84297 & 17.27307792 \\
\hline HS.525922 & 54.111846 & 38.0848143 & 16.027032 & 22.05778262 \\
\hline HCG4 & 52.02505 & 38.0736661 & 13.951384 & 24.12228233 \\
\hline MN1 & 69.20248 & 38.0478432 & 31.154637 & 6.893206166 \\
\hline CLEC1A & 71.985292 & 37.7632783 & 34.222014 & 3.541264242 \\
\hline FAM20A & 77.820807 & 37.729718 & 40.091089 & -2.361371218 \\
\hline$A L D H 2$ & 66.540582 & 37.334502 & 29.20608 & 8.128422392 \\
\hline$E H D 1$ & 52.965111 & 37.1110976 & 15.854014 & 21.257084 \\
\hline NMNAT2 & 63.619487 & 36.2615548 & 27.357932 & 8.903622913 \\
\hline KIAA1199 & 66.223303 & 35.8189545 & 30.404349 & 5.414605574 \\
\hline KLHL13 & 60.59826 & 35.6899059 & 24.908354 & 10.78155167 \\
\hline LEPR & 55.77418 & 35.6854562 & 20.088724 & 15.59673201 \\
\hline SMS & 54.925611 & 35.6405658 & 19.285045 & 16.35552082 \\
\hline JAM2 & 55.792434 & 35.5409367 & 20.251497 & 15.28943996 \\
\hline TOB1 & 74.265798 & 35.5301485 & 38.735649 & -3.205500765 \\
\hline ACSL5 & 60.50137 & 35.3709656 & 25.130404 & 10.24056162 \\
\hline SFRP2 & 75.13541 & 35.116726 & 40.018684 & -4.9019577 \\
\hline UGT3A2 & 49.861588 & 35.060084 & 14.801504 & 20.25858018 \\
\hline HNMT & 54.075316 & 34.9770918 & 19.098224 & 15.87886739 \\
\hline PHLDB2 & 68.831888 & 34.4682469 & 34.363641 & 0.104606126 \\
\hline HS.286666 & 56.068406 & 34.3583383 & 21.710068 & 12.64827032 \\
\hline SELENBP1 & 66.808431 & 34.2560299 & 32.552401 & 1.703628871 \\
\hline TBC1D8 & 63.331815 & 34.0514957 & 29.28032 & 4.771176193 \\
\hline GPR4 & 54.442049 & 34.0008219 & 20.441227 & 13.55959473 \\
\hline$A R L 4 D$ & 46.539182 & 33.3520272 & 13.187155 & 20.164872 \\
\hline SERPINB1 & 59.59758 & 33.2218756 & 26.375704 & 6.846171609 \\
\hline TCN2 & 57.207409 & 33.0428522 & 24.164557 & 8.878295542 \\
\hline FGFRL1 & 53.029408 & 32.958395 & 20.071013 & 12.88738164 \\
\hline MAN1C1 & 55.226112 & 32.514126 & 22.711986 & 9.802139895 \\
\hline OXTR & 62.350785 & 32.0453568 & 30.305428 & 1.739928946 \\
\hline C180RF1 & 53.011275 & 31.9067125 & 21.104562 & 10.80215021 \\
\hline FXYD5 & 47.9475 & 31.5981761 & 16.349324 & 15.24885214 \\
\hline TSC22D1 & 56.535991 & 31.5693995 & 24.966591 & 6.602808354 \\
\hline MBOAT1 & 46.95168 & 31.287494 & 15.664186 & 15.62330761 \\
\hline PDE6A & 45.616977 & 31.2342021 & 14.382775 & 16.85142689 \\
\hline HMHA1 & 57.928421 & 30.8650788 & 27.063342 & 3.801736731 \\
\hline SULF1 & 47.61938 & 30.7694661 & 16.849914 & 13.91955223 \\
\hline CLCNKB & 43.962685 & 30.754402 & 13.208283 & 17.54611929 \\
\hline FAT4 & 46.928609 & 30.740171 & 16.188438 & 14.55173324 \\
\hline CALML4 & 46.71526 & 30.7183496 & 15.99691 & 14.72143933 \\
\hline
\end{tabular}


An oncogenic functional module in meningioma

TABLE 2: Connectivity strength of genes in the oncogenic module* (continued)

\begin{tabular}{|c|c|c|c|c|}
\hline Gene & $\mathrm{k}_{\text {Total }}$ & $\mathrm{k}_{\text {Within }}$ & $\mathrm{k}_{\text {Out }}$ & $k_{\text {Diff }}$ \\
\hline TMEM20 & 51.910543 & 30.521065 & 21.389478 & 9.131586859 \\
\hline CYP4F12 & 47.644483 & 30.0807017 & 17.563781 & 12.51692053 \\
\hline SLC16A9 & 55.309621 & 29.9600739 & 25.349547 & 4.610526902 \\
\hline TP53/11 & 48.91971 & 29.9241109 & 18.995599 & 10.92851171 \\
\hline CHMP1B & 50.517347 & 29.6498198 & 20.867527 & 8.782292372 \\
\hline EYA1 & 50.156311 & 29.0398559 & 21.116455 & 7.923400575 \\
\hline RIPK4 & 41.638271 & 28.8288445 & 12.809427 & 16.01941788 \\
\hline SPTLC3 & 68.877063 & 28.7206109 & 40.156452 & -11.43584107 \\
\hline GADD45B & 56.387845 & 28.5303764 & 27.857468 & 0.672908301 \\
\hline LZTS1 & 74.164723 & 28.0142455 & 46.150477 & -18.13623175 \\
\hline PIK3C2B & 50.789735 & 27.9914468 & 22.798289 & 5.193158155 \\
\hline MET & 55.95372 & 27.8153185 & 28.138402 & -0.32308342 \\
\hline UNC13B & 50.005877 & 27.6928691 & 22.313008 & 5.379861043 \\
\hline ACSF2 & 42.346233 & 27.6105974 & 14.735635 & 12.87496221 \\
\hline C15ORF52 & 47.799629 & 27.5488835 & 20.250745 & 7.298138423 \\
\hline PLCE1 & 53.351366 & 27.3755524 & 25.975814 & 1.39973842 \\
\hline SIX2 & 49.809054 & 27.2042265 & 22.604828 & 4.599398799 \\
\hline TMEM195 & 41.568529 & 26.8311964 & 14.737333 & 12.09386376 \\
\hline$N R 4 A 2$ & 42.088573 & 26.8063421 & 15.282231 & 11.52411149 \\
\hline REEP1 & 52.473201 & 26.7233188 & 25.749882 & 0.973436371 \\
\hline$S H B$ & 44.159135 & 26.6600985 & 17.499036 & 9.161062391 \\
\hline$A D C Y 3$ & 60.796418 & 26.6581086 & 34.13831 & -7.480200999 \\
\hline ASTN2 & 47.361492 & 26.5419923 & 20.8195 & 5.722492236 \\
\hline PGF & 38.778386 & 26.3195466 & 12.45884 & 13.86070701 \\
\hline TNFAIP8L3 & 46.174732 & 26.2606133 & 19.914119 & 6.346494281 \\
\hline CXCR7 & 52.394703 & 25.8960562 & 26.498647 & -0.602590624 \\
\hline SLC20A2 & 53.499574 & 25.6518708 & 27.847703 & -2.195832492 \\
\hline HS6ST2 & 41.850442 & 25.5123381 & 16.338104 & 9.174234209 \\
\hline EYA2 & 57.18162 & 25.2882305 & 31.893389 & -6.605158749 \\
\hline AFAP1 & 45.73445 & 25.0514673 & 20.682983 & 4.368484348 \\
\hline PDE1A & 45.411184 & 25.0329288 & 20.378255 & 4.654673372 \\
\hline MYO5B & 40.078825 & 24.814022 & 15.264803 & 9.549219448 \\
\hline HMGCLL1 & 38.510179 & 24.6394778 & 13.870701 & 10.7687769 \\
\hline SEMA3C & 47.946028 & 24.3910219 & 23.555006 & 0.836016004 \\
\hline$N B L 1$ & 65.594206 & 24.2754579 & 41.318748 & -17.04329014 \\
\hline BCAT2 & 53.43673 & 23.9011265 & 29.535604 & -5.63447704 \\
\hline TEKT3 & 52.68643 & 23.6896794 & 28.996751 & -5.307071562 \\
\hline CCDC34 & 48.151283 & 23.6472239 & 24.504059 & -0.856835355 \\
\hline$A P L P 2$ & 47.080971 & 23.36547 & 23.715501 & -0.350030569 \\
\hline C8ORF34 & 36.91195 & 23.2162621 & 13.695688 & 9.52057455 \\
\hline SLC26A7 & 37.305344 & 23.166268 & 14.139076 & 9.027191859 \\
\hline C10ORF26 & 44.129558 & 23.131567 & 20.997991 & 2.133576376 \\
\hline C2ORF40 & 47.348227 & 22.7171508 & 24.631076 & -1.913925259 \\
\hline$A L X 3$ & 40.123675 & 22.5446289 & 17.579046 & 4.965582441 \\
\hline$A D / 1$ & 37.446839 & 22.4786302 & 14.968209 & 7.51042108 \\
\hline RFTN1 & 42.389419 & 22.4401878 & 19.949231 & 2.490957076 \\
\hline LOC54103 & 42.858358 & 22.3174184 & 20.54094 & 1.776478254 \\
\hline$R R A D$ & 37.159657 & 21.9635591 & 15.196098 & 6.76746107 \\
\hline CYP4X1 & 34.623603 & 21.8296302 & 12.793973 & 9.035657623 \\
\hline
\end{tabular}

(continued) 
X. Chang et al.

TABLE 2: Connectivity strength of genes in the oncogenic module* (continued)

\begin{tabular}{|c|c|c|c|c|}
\hline Gene & $\mathrm{k}_{\text {Total }}$ & $\mathrm{k}_{\text {Within }}$ & $\mathrm{k}_{\text {Out }}$ & $k_{\text {Diff }}$ \\
\hline$K L K 1$ & 36.747332 & 21.7187729 & 15.028559 & 6.69021392 \\
\hline MARCH3 & 32.667551 & 21.4550358 & 11.212515 & 10.24252109 \\
\hline RSPO3 & 35.181944 & 21.4229844 & 13.758959 & 7.664025135 \\
\hline SLC24A3 & 43.296281 & 21.3641068 & 21.932174 & -0.568067224 \\
\hline MICAL1 & 40.462948 & 21.2879353 & 19.175012 & 2.112922943 \\
\hline TRPC3 & 32.613814 & 21.1406533 & 11.473161 & 9.667492816 \\
\hline FLJ20920 & 33.063828 & 20.9677145 & 12.096113 & 8.871601067 \\
\hline ZNF680 & 45.761023 & 20.8070623 & 24.95396 & -4.146897924 \\
\hline CLCNKA & 32.411415 & 20.6696875 & 11.741727 & 8.927960491 \\
\hline ANO2 & 30.642068 & 20.6683747 & 9.973693 & 10.6946812 \\
\hline C10ORF33 & 35.261246 & 20.6632203 & 14.598025 & 6.065194948 \\
\hline PDE5A & 34.443581 & 20.6569813 & 13.786599 & 6.870382082 \\
\hline$S Q R D L$ & 38.790877 & 20.5781296 & 18.212747 & 2.365382227 \\
\hline CTSZ & 36.013168 & 20.5570576 & 15.456111 & 5.100946672 \\
\hline PROS1 & 45.22208 & 20.3650076 & 24.857072 & -4.492064393 \\
\hline LOC342979 & 38.78767 & 20.3113441 & 18.476326 & 1.835018433 \\
\hline NCAPD3 & 49.296213 & 20.2177589 & 29.078454 & -8.860695375 \\
\hline AFAP1L2 & 42.80353 & 20.1962619 & 22.607268 & -2.411006256 \\
\hline NPTX2 & 39.77959 & 20.0275871 & 19.752003 & 0.27558387 \\
\hline BAIAP2L2 & 37.365078 & 19.8812506 & 17.483828 & 2.397422803 \\
\hline C10ORF73 & 35.715635 & 19.7470922 & 15.968542 & 3.778549776 \\
\hline PCOLCE2 & 34.435991 & 19.6626869 & 14.773304 & 4.889382806 \\
\hline SLITRK4 & 36.202406 & 19.4122635 & 16.790143 & 2.622120752 \\
\hline TGFBR3 & 47.362059 & 19.3067906 & 28.055268 & -8.748477346 \\
\hline BMP5 & 33.001158 & 19.2339653 & 13.767193 & 5.466772139 \\
\hline CHST4 & 39.852826 & 19.1641381 & 20.688687 & -1.524549272 \\
\hline ITM2C & 48.764021 & 19.0204147 & 29.743606 & -10.72319115 \\
\hline PSAT1 & 45.139185 & 18.9106324 & 26.228553 & -7.31792061 \\
\hline ELOVL6 & 36.392406 & 18.8322914 & 17.560115 & 1.272176728 \\
\hline IGFBP3 & 32.753029 & 18.7525297 & 14.000499 & 4.75203061 \\
\hline CTSK & 58.11487 & 18.5960699 & 39.5188 & -20.92273007 \\
\hline$B O C$ & 36.28372 & 18.2785785 & 18.005141 & 0.273437118 \\
\hline C10ORF11 & 41.198815 & 18.1827797 & 23.016035 & -4.833255754 \\
\hline $\mathrm{CDH} 1$ & 33.942365 & 18.1071814 & 15.835183 & 2.271998064 \\
\hline ST3GAL5 & 33.779051 & 18.1022425 & 15.676808 & 2.425434052 \\
\hline IL34 & 30.49039 & 18.0939202 & 12.39647 & 5.697450237 \\
\hline NELL2 & 45.247855 & 17.9842398 & 27.263615 & -9.279375304 \\
\hline GPR137C & 43.825082 & 17.9615367 & 25.863546 & -7.902008965 \\
\hline SFRP1 & 27.271184 & 17.8838074 & 9.387377 & 8.496430893 \\
\hline LOC728211 & 27.579571 & 17.7838233 & 9.795747 & 7.988075957 \\
\hline DEFB1 & 38.236686 & 17.4005093 & 20.836176 & -3.435667055 \\
\hline ASAM & 46.900685 & 17.3110555 & 29.58963 & -12.27857443 \\
\hline SHF & 26.368082 & 16.9777555 & 9.390326 & 7.587428967 \\
\hline COPZ2 & 48.25746 & 16.801448 & 31.456012 & -14.65456387 \\
\hline PLD1 & 32.6877 & 16.7055927 & 15.982108 & 0.723485181 \\
\hline BTG2 & 31.200689 & 16.4685995 & 14.73209 & 1.736509694 \\
\hline STARD8 & 28.945853 & 16.2884431 & 12.65741 & 3.631033206 \\
\hline $\mathrm{CHSY} 3$ & 27.143499 & 16.2329674 & 10.910532 & 5.322435723 \\
\hline FOX01 & 31.700055 & 16.1117571 & 15.588298 & 0.5234588878 \\
\hline
\end{tabular}

(continued) 
An oncogenic functional module in meningioma

TABLE 2: Connectivity strength of genes in the oncogenic module* (continued)

\begin{tabular}{|c|c|c|c|c|}
\hline Gene & $\mathrm{k}_{\text {Total }}$ & $k_{\text {Within }}$ & $\mathrm{k}_{\text {Out }}$ & $k_{\text {Diff }}$ \\
\hline MPZL2 & 28.159703 & 16.0215742 & 12.138129 & 3.883445349 \\
\hline LOC388494 & 28.64752 & 15.9095065 & 12.738013 & 3.171493282 \\
\hline TMEM16B & 22.768178 & 15.653603 & 7.114575 & 8.539027983 \\
\hline FAM63B & 32.989171 & 15.6484054 & 17.340766 & -1.692360696 \\
\hline CD55 & 26.193048 & 15.6275422 & 10.565506 & 5.062036543 \\
\hline AHNAK2 & 33.934328 & 15.6012845 & 18.333044 & -2.731759213 \\
\hline POL3S & 33.010494 & 15.5574662 & 17.453027 & -1.895561148 \\
\hline PDGFRL & 38.28427 & 15.3895187 & 22.894751 & -7.505232417 \\
\hline C140RF78 & 31.374417 & 15.1415776 & 16.232839 & -1.091261493 \\
\hline DACT1 & 27.437047 & 14.924693 & 12.512354 & 2.412339487 \\
\hline COL18A1 & 26.598642 & 14.6509657 & 11.947677 & 2.703289168 \\
\hline PTPN13 & 28.144953 & 14.3940342 & 13.750919 & 0.643115561 \\
\hline FOXP1 & 31.035432 & 14.2924979 & 16.742934 & -2.450436567 \\
\hline SETBP1 & 28.754646 & 14.290703 & 14.463943 & -0.173240412 \\
\hline MIR185 & 30.751939 & 14.2429239 & 16.509015 & -2.266090945 \\
\hline PPFIBP1 & 38.216457 & 14.1651799 & 24.051277 & -9.886097078 \\
\hline PVRL3 & 45.43931 & 14.0844661 & 31.354843 & -17.27037735 \\
\hline ADAMTSL1 & 26.517671 & 14.0231056 & 12.494565 & 1.528540236 \\
\hline EMILIN1 & 26.868861 & 13.9034047 & 12.965456 & 0.937948195 \\
\hline TMEM30B & 28.077249 & 13.8631885 & 14.214061 & -0.35087206 \\
\hline LOC647979 & 24.725424 & 13.8270804 & 10.898344 & 2.928736384 \\
\hline$F 10$ & 22.97621 & 13.6589004 & 9.317309 & 4.341591077 \\
\hline CYP3A5 & 26.122458 & 13.5492804 & 12.573178 & 0.976102708 \\
\hline RERG & 37.786428 & 13.3516863 & 24.434741 & -11.08305495 \\
\hline KLHL14 & 19.19865 & 13.2802935 & 5.918357 & 7.361936841 \\
\hline GMPR & 25.509252 & 13.2028389 & 12.306413 & 0.896425719 \\
\hline$K L F 4$ & 37.574339 & 13.1700615 & 24.404277 & -11.23421574 \\
\hline TMEM37 & 24.225877 & 13.0406309 & 11.185246 & 1.855385222 \\
\hline ZNF503 & 28.288625 & 12.9115998 & 15.377025 & -2.465425451 \\
\hline FAM101A & 18.584357 & 12.8186932 & 5.765664 & 7.053029009 \\
\hline C110RF80 & 30.37899 & 12.7866418 & 17.592348 & -4.805706326 \\
\hline ATOH8 & 19.697787 & 12.7577426 & 6.940044 & 5.81769829 \\
\hline C10ORF65 & 22.300592 & 12.6956716 & 9.604921 & 3.090751068 \\
\hline TAGLN & 28.571186 & 12.6764854 & 15.8947 & -3.218215036 \\
\hline EDG7 & 24.217116 & 12.6736048 & 11.543511 & 1.130093344 \\
\hline CTHRC1 & 35.16698 & 12.5428042 & 22.624176 & -10.08137184 \\
\hline RASEF & 17.300335 & 12.477871 & 4.822464 & 7.655406849 \\
\hline SALL4 & 21.862568 & 12.3921531 & 9.470415 & 2.921738591 \\
\hline C110RF70 & 26.184607 & 12.2370312 & 13.947576 & -1.710544996 \\
\hline AGPAT9 & 22.629747 & 12.0724255 & 10.557322 & 1.515103936 \\
\hline SERTAD4 & 21.015614 & 12.0376522 & 8.977961 & 3.059690825 \\
\hline NPNT & 29.061401 & 11.7260254 & 17.335375 & -5.609350123 \\
\hline INMT & 20.773939 & 11.723038 & 9.050901 & 2.672137019 \\
\hline B4GALT1 & 28.03345 & 11.5831866 & 16.450263 & -4.867076274 \\
\hline SMAD6 & 24.775931 & 11.5825681 & 13.193362 & -1.610794394 \\
\hline$A N L N$ & 38.970357 & 11.5347278 & 27.43563 & -15.90090182 \\
\hline NTN3 & 20.823662 & 11.4167512 & 9.406911 & 2.009840221 \\
\hline PBX3 & 21.974249 & 11.3341401 & 10.640109 & 0.694030737 \\
\hline C1ORF133 & 21.208224 & 11.321314 & 9.88691 & 1.434403989 \\
\hline
\end{tabular}

(continued) 
X. Chang et al.

TABLE 2: Connectivity strength of genes in the oncogenic module ${ }^{*}$ (continued)

\begin{tabular}{|c|c|c|c|c|}
\hline Gene & $\mathrm{k}_{\text {Total }}$ & $\mathrm{k}_{\text {Within }}$ & $\mathrm{k}_{\text {Out }}$ & $\mathrm{k}_{\text {Diff }}$ \\
\hline SRGAP1 & 23.163626 & 11.2586245 & 11.905002 & -0.646377281 \\
\hline$B O P 1$ & 33.917157 & 11.1905378 & 22.726619 & -11.53608163 \\
\hline FREM2 & 20.97208 & 10.8616073 & 10.110473 & 0.751134643 \\
\hline$R H O B$ & 20.903824 & 10.8321524 & 10.071672 & 0.760480515 \\
\hline ANO6 & 41.978064 & 10.6445366 & 31.333528 & -20.68899102 \\
\hline TJP3 & 23.072419 & 10.601802 & 12.470617 & -1.868814754 \\
\hline FGF11 & 23.884247 & 10.5932122 & 13.291034 & -2.697822252 \\
\hline ARHGAP10 & 23.397384 & 10.563653 & 12.833731 & -2.270077847 \\
\hline HES1 & 44.486825 & 10.541474 & 33.945351 & -23.40387648 \\
\hline NQ01 & 22.690692 & 10.5388687 & 12.151824 & -1.612955021 \\
\hline LOC387882 & 28.96743 & 10.5362801 & 18.43115 & -7.89486971 \\
\hline RPS6KA5 & 34.274251 & 10.5287587 & 23.745492 & -13.21673371 \\
\hline$K L K 7$ & 19.826662 & 10.5058015 & 9.320861 & 1.184940615 \\
\hline MEOX2 & 24.262074 & 10.2859715 & 13.976103 & -3.690131443 \\
\hline MND1 & 32.638701 & 10.1533671 & 22.485333 & -12.33196635 \\
\hline EDN1 & 28.699333 & 10.0883972 & 18.610936 & -8.52253882 \\
\hline$S I X 1$ & 26.219793 & 10.0049291 & 16.214864 & -6.209935026 \\
\hline HSPB6 & 34.07586 & 9.9289733 & 24.146886 & -14.21791312 \\
\hline$F G R$ & 19.339602 & 9.9195676 & 9.420034 & 0.499533463 \\
\hline SMOX & 26.819625 & 9.9041951 & 16.91543 & -7.01123496 \\
\hline$D S P$ & 19.183297 & 9.8944749 & 9.288822 & 0.605652459 \\
\hline NFKBIZ & 17.576362 & 9.8651966 & 7.711165 & 2.154031451 \\
\hline$C D C 7$ & 19.695647 & 9.8613918 & 9.834256 & 0.027136139 \\
\hline $\mathrm{CDH} 5$ & 15.342002 & 9.7681584 & 5.573844 & 4.194314517 \\
\hline S100P & 16.638205 & 9.6795362 & 6.958669 & 2.720867288 \\
\hline MR1 & 23.281125 & 9.6457053 & 13.63542 & -3.989714456 \\
\hline KCNK5 & 14.699684 & 9.6202299 & 5.079454 & 4.540775581 \\
\hline LPAR3 & 17.438678 & 9.5833715 & 7.855307 & 1.728064961 \\
\hline FZD7 & 19.926706 & 9.5367574 & 10.389948 & -0.853190853 \\
\hline FABP3 & 22.269481 & 9.5310268 & 12.738454 & -3.207427529 \\
\hline COL23A1 & 20.185272 & 9.4171492 & 10.768122 & -1.350973071 \\
\hline ZBTB16 & 20.485859 & 9.2975164 & 11.188343 & -1.890826658 \\
\hline PDZD2 & 35.435857 & 9.2492791 & 26.186577 & -16.93729831 \\
\hline STOX1 & 35.103324 & 9.0969995 & 26.006324 & -16.90932479 \\
\hline C10ORF105 & 21.291921 & 8.9998536 & 12.292067 & -3.292213634 \\
\hline RNF24 & 18.454095 & 8.9591057 & 9.494989 & -0.535883418 \\
\hline C1ORF21 & 48.734478 & 8.8720507 & 39.862427 & -30.99037668 \\
\hline$C F B$ & 27.560248 & 8.8657687 & 18.69448 & -9.828710926 \\
\hline CHODL & 16.820116 & 8.5512157 & 8.268901 & 0.282315188 \\
\hline GCGR & 13.388728 & 8.3906571 & 4.998071 & 3.392586019 \\
\hline LOC100133551 & 13.771589 & 8.2338936 & 5.537696 & 2.696197965 \\
\hline RCSD1 & 14.070933 & 8.1794294 & 5.891504 & 2.287925604 \\
\hline GDE1 & 20.05449 & 8.1312334 & 11.923257 & -3.792023541 \\
\hline HRCT1 & 17.205117 & 8.0024474 & 9.202669 & -1.200221661 \\
\hline GALNTL4 & 18.831225 & 7.8499182 & 10.981307 & -3.131388378 \\
\hline PRDM6 & 20.245629 & 7.836219 & 12.40941 & -4.573191005 \\
\hline SLC25A29 & 15.692794 & 7.4481252 & 8.244669 & -0.796544006 \\
\hline TIMP3 & 13.91059 & 7.4429861 & 6.467604 & 0.975382132 \\
\hline$K L K 8$ & 18.009085 & 7.3627909 & 10.646294 & -3.283503569 \\
\hline
\end{tabular}

(continued) 
An oncogenic functional module in meningioma

TABLE 2: Connectivity strength of genes in the oncogenic module* (continued)

\begin{tabular}{|c|c|c|c|c|}
\hline Gene & $\mathrm{k}_{\text {Total }}$ & $\mathrm{k}_{\text {Within }}$ & $\mathrm{k}_{\text {Out }}$ & $k_{\text {Diff }}$ \\
\hline SLC22A18 & 14.091617 & 7.2046128 & 6.887005 & 0.317608148 \\
\hline $\mathrm{CHCHD} 6$ & 17.018761 & 6.9436167 & 10.075144 & -3.131527701 \\
\hline FGF2 & 18.014674 & 6.9053371 & 11.109337 & -4.203999999 \\
\hline LOC388588 & 15.042224 & 6.7182982 & 8.323926 & -1.60562803 \\
\hline TCF3 & 19.122877 & 6.5475548 & 12.575322 & -6.027767481 \\
\hline GALNT12 & 11.082285 & 6.4959277 & 4.586357 & 1.909570346 \\
\hline SLC13A4 & 13.149774 & 6.3012236 & 6.848551 & -0.547327043 \\
\hline$X Y L T 1$ & 11.225407 & 6.2435902 & 4.981817 & 1.26177294 \\
\hline ENPP1 & 14.339533 & 6.2405762 & 8.098957 & -1.858380392 \\
\hline MSLN & 12.863767 & 6.2334341 & 6.630333 & -0.396899349 \\
\hline HS. 572444 & 13.658788 & 6.2107538 & 7.448034 & -1.237280446 \\
\hline$A O X 1$ & 17.079673 & 6.1897817 & 10.889891 & -4.700109256 \\
\hline SLC6A20 & 9.677573 & 6.164331 & 3.513242 & 2.651088869 \\
\hline IL18R1 & 13.32097 & 6.1067296 & 7.214241 & -1.107511012 \\
\hline PRG4 & 11.906875 & 6.0772185 & 5.829657 & 0.24756167 \\
\hline PLXNA2 & 12.0217 & 5.8286637 & 6.193036 & -0.364372752 \\
\hline MST4 & 13.770683 & 5.7500375 & 8.020646 & -2.270608299 \\
\hline CYB5R2 & 16.525844 & 5.7451343 & 10.78071 & -5.035575398 \\
\hline SPATA18 & 14.322105 & 5.7384282 & 8.583677 & -2.845248513 \\
\hline NXPH3 & 8.373418 & 5.6906602 & 2.682758 & 3.007902253 \\
\hline FM01 & 9.657889 & 5.47014 & 4.187749 & 1.282391373 \\
\hline C6ORF26 & 10.18049 & 5.4453886 & 4.735101 & 0.710287393 \\
\hline GADL1 & 12.256558 & 5.3158141 & 6.940744 & -1.624930024 \\
\hline CLDN7 & 9.533733 & 5.1559934 & 4.377739 & 0.778254026 \\
\hline AXUD1 & 10.304096 & 5.1110176 & 5.193079 & -0.082061128 \\
\hline GINS4 & 26.081854 & 4.9081524 & 21.173701 & -16.26554896 \\
\hline LHCGR & 8.31711 & 4.884274 & 3.432836 & 1.451438378 \\
\hline FRZB & 11.067715 & 4.8388184 & 6.228896 & -1.390077976 \\
\hline FLJ30375 & 9.323856 & 4.7718644 & 4.551992 & 0.219872435 \\
\hline TNFRSF21 & 8.721926 & 4.7517213 & 3.970204 & 0.78151713 \\
\hline C12ORF48 & 20.386633 & 4.7285983 & 15.658035 & -10.92943636 \\
\hline CCDC68 & 12.182918 & 4.6767419 & 7.506176 & -2.829434386 \\
\hline FANCC & 22.845453 & 4.4256789 & 18.419774 & -13.99409526 \\
\hline HS.444785 & 9.280858 & 4.3534548 & 4.927403 & -0.57394811 \\
\hline LPHN2 & 11.426724 & 4.3128621 & 7.113862 & -2.8010001 \\
\hline NAPSB & 8.7574 & 4.2768968 & 4.480503 & -0.203605997 \\
\hline СРXM2 & 10.082331 & 4.2752754 & 5.807055 & -1.531780019 \\
\hline HS.119933 & 10.118276 & 4.2438622 & 5.874414 & -1.630551804 \\
\hline MY01B & 8.213893 & 4.1076496 & 4.106243 & 0.001406504 \\
\hline MLF1IP & 20.299115 & 4.0235208 & 16.275595 & -12.25207393 \\
\hline LYPLAL1 & 8.038086 & 3.9962114 & 4.041875 & -0.045663261 \\
\hline MFAP2 & 16.584184 & 3.7779842 & 12.8062 & -9.028215801 \\
\hline CLIC3 & 9.925067 & 3.7155352 & 6.209532 & -2.493996661 \\
\hline$A B C G 2$ & 11.450555 & 3.6594262 & 7.791129 & -4.131703004 \\
\hline ENG & 10.649239 & 3.5811315 & 7.068107 & -3.486975962 \\
\hline COL8A2 & 9.139634 & 3.5778818 & 5.561753 & -1.983870823 \\
\hline LYPD6B & 6.694317 & 3.4947409 & 3.199576 & 0.295165126 \\
\hline ANGPTL7 & 9.985216 & 3.3629758 & 6.62224 & -3.259264177 \\
\hline GPSM2 & 16.000406 & 3.3471303 & 12.653276 & -9.306145687 \\
\hline
\end{tabular}

(continued) 
TABLE 2: Connectivity strength of genes in the oncogenic module* (continued)

\begin{tabular}{lcccc}
\hline \multicolumn{1}{c}{ Gene } & $\mathrm{k}_{\text {Total }}$ & $\mathrm{k}_{\text {within }}$ & $\mathrm{k}_{\text {Out }}$ & $\mathrm{k}_{\text {Diff }}$ \\
\hline SIX4 & 21.397128 & 3.2923045 & 18.104824 & -14.81251918 \\
ZBTB7C & 9.063249 & 3.2155378 & 5.847711 & -2.632172957 \\
PRRX1 & 6.612249 & 3.1330223 & 3.479227 & -0.346204832 \\
TMEM154 & 10.302565 & 2.9347514 & 7.367814 & -4.433062307 \\
COL12A1 & 10.96393 & 2.9258159 & 8.038115 & -5.11229867 \\
HS.575038 & 6.718715 & 2.8289754 & 3.88974 & -1.060764291 \\
MSX1 & 4.599336 & 2.4443386 & 2.154997 & 0.289341444 \\
PLEKHG3 & 7.857878 & 1.9532737 & 5.904604 & -3.951330301 \\
ST3GAL6 & 8.276674 & 1.7560559 & 6.520618 & -4.764561802 \\
CD177 & 3.733092 & 1.5403244 & 2.192767 & -0.652442683 \\
PPP1R3C & 3.524628 & 1.4846765 & 2.039952 & -0.555275244 \\
FAM107B & 4.811333 & 1.3696313 & 3.441702 & -2.072070786 \\
ADAMTSL5 & 2.497067 & 0.6625621 & 1.834505 & -1.17194317 \\
\hline
\end{tabular}

${ }^{*} \mathrm{k}_{\text {Total }}=$ total connectivity; $\mathrm{k}_{\text {Within }}=$ connectivity within the oncogenic module; $\mathrm{k}_{\text {Out }}=$ connectivity outside the oncogenic module; $\mathrm{k}_{\text {Diff }}$ $=$ difference between $\mathrm{k}_{\text {within }}$ and $\mathrm{k}_{\text {out }}$.

$\dagger$ Important hubs that are addressed in the text.

and early step in meningioma pathogenesis. Nearly half of our samples harbored significant deletions in chromosome $22 \mathrm{q}$, a finding that substantiates previous reports. ${ }^{23,40}$ Loss of chromosome 22, however, was not apparent in all of the malignant samples; indeed only 2 of 4 exhibited deletion in this region. Loss of chromosome 4 was evident in 3 of 4 malignant samples, suggesting a potential role in malignant transformation. One malignant sample (Sample ID 254) demonstrated only minor changes in copy number across the entire genome. The sole chromosomal abnormality noted in this tumor was the gain of chromosome 21 (Fig. 1). Although these observations

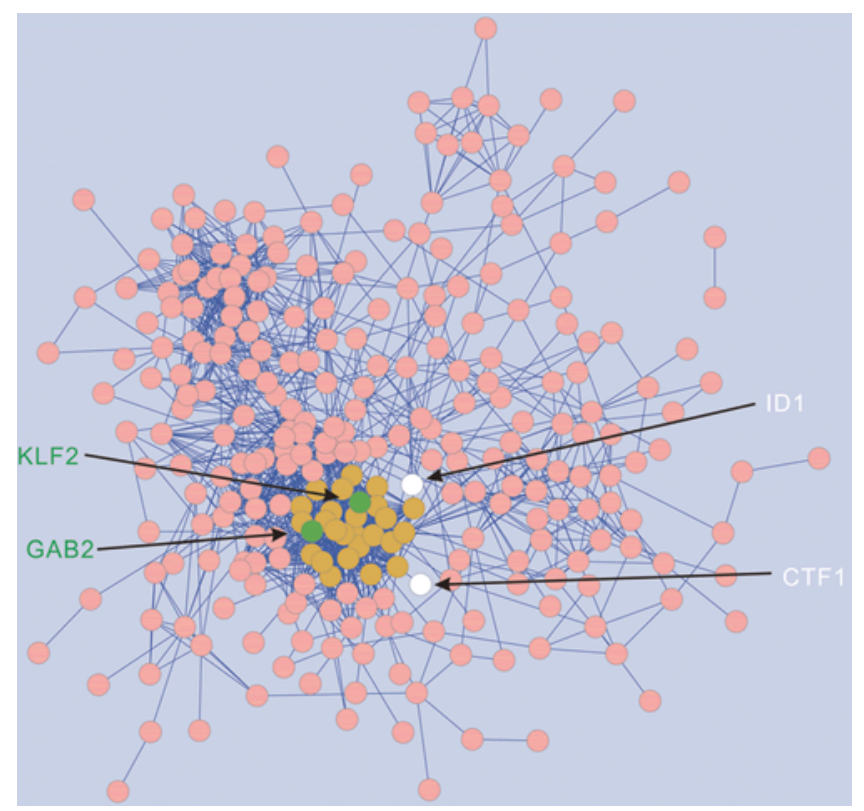

FIG. 8. Network topology of the oncogenic module. The core region is marked by yellow, hub genes within the core region are marked in green, and hub genes outside the core region are marked in white. support a role for chromosomal-scale abnormalities in the development and progression of meningiomas, they reaffirm previously known challenges in extrapolating complicated mechanisms from a relatively small sample size.

We further investigated gene expression differences between nonmalignant and malignant meningiomas at the transcriptome level. The whole-genome expression profile was consistent among the nonmalignant (benign and atypical) samples. These patterns, however, were altered in the malignant samples, suggesting disruption of normal gene regulatory mechanisms. Furthermore, each

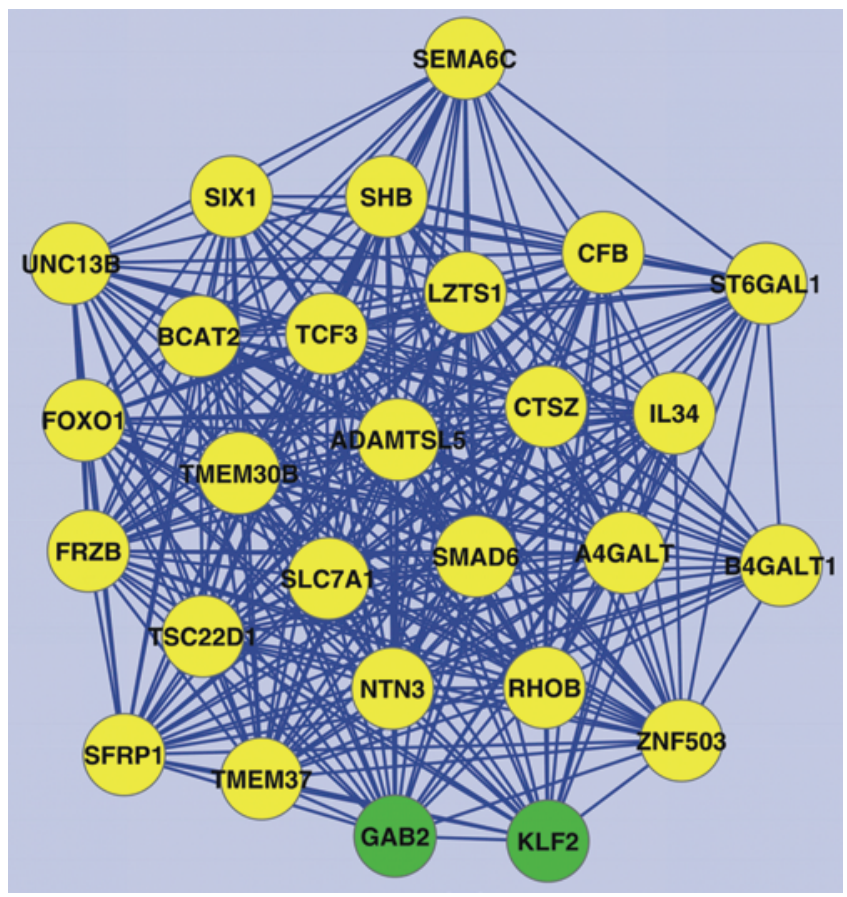

FIG. 9. The network topology of the core region; hub genes within the core region are marked in green. 
malignant sample presented a unique expression pattern, implying heterogeneity in the factors involved with alterations in the gene regulatory system (Fig. 2). Additionally, based on analysis of previously identified abnormal signaling pathways in meningiomas, we found that genes such as $M N 1$ and bone morphogenetic proteins are also differentially expressed between benign and malignant meningiomas.

We also identified some genes that displayed a high association between DNA copy number and gene expression. These genes were enriched in chromosome 1,2, 3, 4,16 , and 22 , which is consistent with regions harboring high levels of copy number alterations. As previously stated, loss of chromosome 22 was identified in the majority of samples, whereas loss of chromosome 4 was noted in 3 of 4 malignant meningiomas. Copy number alterations may therefore exert a dose-dependent effect on gene expression level.

Since biological network studies provided systematical understanding of the biological process, we also applied WGCNA to identify gene coexpression modules and potential oncogenes., ${ }^{2,6,45}$ The WGCNA approach has been widely used to identify potential oncogenic coexpression modules and predict the molecular targets and prognosis markers. ${ }^{14,16,38,55,58}$ For instance, Horvath et al. have successfully identified the gene $A S P M$ as a molecular target for glioblastoma, another primary brain tumor. ${ }^{14}$ Ivliev et al. also identified transcriptional modules related to proastrocytic differentiation and sprout signaling in gliomas. ${ }^{16}$ In our study, among the 23 coexpression modules, we identified one that was strongly associated with tumorigenesis and could be considered an oncogenic module. This module contains 356 genes and is enriched in glycoprotein/membrane/blood vessel development and cell migration. We also identified the intramodular hub genes that are thought to contribute significantly to the topological architecture and biological process of functional modules. Four oncogene hubs $(G A B 2, K L F 2, I D 1$, and $C T F 1$ ) were detected among the top 15 most connected genes within the oncogenic module. Prior studies have suggested that these 4 genes are associated with leukemia. ${ }^{25,37,52,54}$ Interestingly, the meningioma 1 gene, often abbreviated as $M N I$, was also identified in this oncogenic module. MN1 was first discovered in 1995 from a meningioma patient with a balanced translocation that disrupts $M N 1$ in its first exon. In this case, no expression of MN1 mRNA was observed, so MNI was considered as a candidate tumor suppressor gene, affecting meningioma formation via inactivation. ${ }^{24}$ However, further research has suggested that overexpression of the $M N 1$ gene can induce acute myeloid leukemia. Our study demonstrated differential MNI gene expression between benign and malignant meningiomas. Other differentially expressed oncogenes found in this module were IGFBP5, $M E T$, and TGFBR3. IGFBP5 is also upregulated by MN1 and linked to other cancer types, including glioblastoma multiforme. ${ }^{31,49}$

We further identified a core region within the oncogenic coexpression module, consisting of the hub genes GAB2 and KLF2. The additional hub genes IDI and $C T F 1$ were directly connected to these members of the core region. Although $M N 1$ was not directly connected in the coexpression network, protein-protein interaction networks show that $M N 1$ can connect to $K L F 2$ via $E P 300$. These results suggest that $G A B 2$ and $K L F 2$ may play an important role in the meningioma developmental pathway. Oncogenes interacting with $G A B 2$ and $K L F 2$ can function together as a small group. In turn, oncogenes indirectly connected to $G A B 2$ and $K L F 2$ via neighbors can form a large oncogenic module for meningiomas.

Considering the small sample size, we analyzed 2 relatively large gene expression data sets including 68 and 56 meningioma samples, respectively, although the number of malignant samples is still quite small (6 and 3 , respectively). Functional enrichment analysis indicated that our data (the USC study) and those from the Scheck study were highly consistent with the Cancer Genome Anatomy Project database. We also applied WGCNA to these 2 data sets. However, WGCNA failed to find a significant meningioma-related module. A possible reason may be the imbalance of sample proportion from different subtypes because only 6 malignant samples of the total 68 samples are involved in the UCLA study and only 3 malignant samples of the total 56 samples are involved in the Scheck data set. This issue will lead to a bias in calculating the expression correlation between gene pairs. Therefore, the relative proportion of subtypes may be more important than sample size when applying WGCNA to construct gene coexpression networks.

Although limited by sample size, this systematic analysis of genomic and transcriptome differences between benign and malignant meningiomas revealed novel insight into the machinery of meningioma pathogenesis and transformation. If confirmed in larger studies, these alterations may serve as targets for novel diagnostic modalities and therapeutic interventions.

\section{Conclusions}

In summary, copy number alteration analysis confirmed the high frequency of chromosome 22 monosomy and the association between WHO grade and chromosomal abnormalities. Transcriptome analysis identified several genes that were highly differentially expressed between the benign and malignant groups, including a meningioma candidate gene, $M N 1$. In addition, we used a weighted gene coexpression network analysis and identified an oncogenic module associated with meningiomas. Hub oncogenes in this module are also associated with the development of leukemia, implying potential common pathways between the 2 types of neoplastic diseases.

\section{Acknowledgment}

We thank members of the Wang lab for helpful comments and suggestions on the analytical strategies.

\section{Disclosure}

This project was supported in part by grant IRG-58-007-51 from the American Cancer Society to K.W., and in part by Southern California Clinical and Translational Science Institute (NIH/NCRR/ NCATS) through grant KL2RR031991. The authors declare that they have no competing interests. 


\section{Chang et al.}

Author contributions to the study and manuscript preparation include the following. Conception and design: Mack, Zada, Wang. Acquisition of data: Gao, Russin, Zeng, He, Giannotta, Zada. Analysis and interpretation of data: Chang, Shi, Gao, Weisenberger, Zada, Wang. Drafting the article: Chang, He, Wang. Critically revising the article: Mack, Russin, Zada, Wang. Reviewed submitted version of manuscript: Mack, Zada, Wang. Approved the final version of the manuscript on behalf of all authors: Mack. Statistical analysis: Chang, Shi, Weisenberger, Wang. Administrative/technical/material support: Chen, Wang. Study supervision: Mack, Chen, Giannotta, Wang.

\section{References}

1. Bader GD, Hogue CW: An automated method for finding molecular complexes in large protein interaction networks. BMC Bioinformatics 4:2, 2003

2. Barabási AL, Oltvai ZN: Network biology: understanding the cell's functional organization. Nat Rev Genet 5:101-113, 2004

3. Batistatou A, Charalabopoulos AK, Scopa CD, Nakanishi Y, Kappas A, Hirohashi S, et al: Expression patterns of dysadherin and E-cadherin in lymph node metastases of colorectal carcinoma. Virchows Arch 448:763-767, 2006

4. Beroukhim R, Getz G, Nghiemphu L, Barretina J, Hsueh T, Linhart D, et al: Assessing the significance of chromosomal aberrations in cancer: methodology and application to glioma. Proc Natl Acad Sci U S A 104:20007-20012, 2007

5. Chang X, Liu S, Yu YT, Li YX, Li YY: Identifying modules of coexpressed transcript units and their organization of Saccharopolyspora erythraea from time series gene expression profiles. PLoS ONE 5:e12126, 2010

6. Chang X, Wang Z, Hao P, Li YY, Li YX: Exploring mitochondrial evolution and metabolism organization principles by comparative analysis of metabolic networks. Genomics 95: 339-344, 2010

7. Diskin SJ, Li M, Hou C, Yang S, Glessner J, Hakonarson H, et al: Adjustment of genomic waves in signal intensities from whole-genome SNP genotyping platforms. Nucleic Acids Res 36:e126, 2008

8. Dobbins SE, Broderick P, Melin B, Feychting M, Johansen $\mathrm{C}$, Andersson U, et al: Common variation at $10 \mathrm{p} 12.31$ near MLLT10 influences meningioma risk. Nat Genet 43:825827,2011

9. Emilsson V, Thorleifsson G, Zhang B, Leonardson AS, Zink F, Zhu J, et al: Genetics of gene expression and its effect on disease. Nature 452:423-428, 2008

10. Fèvre-Montange M, Champier J, Durand A, Wierinckx A, Honnorat J, Guyotat J, et al: Microarray gene expression profiling in meningiomas: differential expression according to grade or histopathological subtype. Int J Oncol 35:13951407,2009

11. Gao F, Shi L, Russin J, Zeng L, Chang X, He S, et al: DNA methylation in the malignant transformation of meningiomas. PLoS ONE 8:e54114, 2013

12. Giusti M, Bocca L, Florio T, Corsaro A, Spaziante R, Schettini $\mathrm{G}$, et al: In vitro effect of human recombinant leptin and expression of leptin receptors on growth hormone-secreting human pituitary adenomas. Clin Endocrinol (Oxf) 57:449455,2002

13. Han JD, Bertin N, Hao T, Goldberg DS, Berriz GF, Zhang LV, et al: Evidence for dynamically organized modularity in the yeast protein-protein interaction network. Nature 430:88-93, 2004

14. Horvath S, Zhang B, Carlson M, Lu KV, Zhu S, Felciano RM, et al: Analysis of oncogenic signaling networks in glioblastoma identifies ASPM as a molecular target. Proc Natl Acad Sci U S A 103:17402-17407, 2006

15. Huang W, Sherman BT, Lempicki RA: Systematic and inte- grative analysis of large gene lists using DAVID bioinformatics resources. Nat Protoc 4:44-57, 2009

16. Ivliev AE, 't Hoen PA, Sergeeva MG: Coexpression network analysis identifies transcriptional modules related to proastrocytic differentiation and sprouty signaling in glioma. Cancer Res 70:10060-10070, 2010

17. Jayapandian M, Chapman A, Tarcea VG, Yu C, Elkiss A, Ianni A, et al: Michigan Molecular Interactions (MiMI): putting the jigsaw puzzle together. Nucleic Acids Res 35 (Database issue):D566-D571, 2007

18. Keller A, Ludwig N, Backes C, Romeike BF, Comtesse N, Henn W, et al: Genome wide expression profiling identifies specific deregulated pathways in meningioma. Int $\mathbf{J}$ Cancer 124:346-351, 2009

19. Kraft R, Krause P, Jung S, Basrai D, Liebmann L, Bolz J, et al: BK channel openers inhibit migration of human glioma cells. Pflugers Arch 446:248-255, 2003

20. Kristiansen MT, Clausen LR, Nielsen S, Blaabjerg O, Ledet T, Rasmussen LM, et al: Expression of leptin receptor isoforms and effects of leptin on the proliferation and hormonal secretion in human pituitary adenomas. Horm Res 62:129-136, 2004

21. Langfelder P, Horvath S: WGCNA: an R package for weighted correlation network analysis. BMC Bioinformatics 9:559, 2008

22. Lee JY, Finkelstein S, Hamilton RL, Rekha R, King JT Jr, Omalu B: Loss of heterozygosity analysis of benign, atypical, and anaplastic meningiomas. Neurosurgery 55:1163-1173, 2004

23. Lee Y, Liu J, Patel S, Cloughesy T, Lai A, Farooqi H, et al: Genomic landscape of meningiomas. Brain Pathol 20:751-762, 2010

24. Lekanne Deprez RH, Riegman PH, Groen NA, Warringa UL, van Biezen NA, Molijn AC, et al: Cloning and characterization of MN1, a gene from chromosome 22q11, which is disrupted by a balanced translocation in a meningioma. Oncogene 10:1521-1528, 1995

25. Lister J, Forrester WC, Baron MH: Inhibition of an erythroid differentiation switch by the helix-loop-helix protein Id1. J Biol Chem 270:17939-17946, 1995

26. Liu T, Jankovic D, Brault L, Ehret S, Baty F, Stavropoulou V, et al: Functional characterization of high levels of meningioma 1 as collaborating oncogene in acute leukemia. Leukemia 24:601-612, 2010

27. Liu X, Chang Y, Reinhart PH, Sontheimer H, Chang Y: Cloning and characterization of glioma BK, a novel BK channel isoform highly expressed in human glioma cells. J Neurosci 22:1840-1849, 2002

28. Louis DN, Scheithauer BW, Budka H, von Deimling A, Kepes JJ: Meningiomas, in Kleihues P, Cavenee WK (eds): World Health Organization Classification of Tumours. Pathology and Genetics: Tumours of the Nervous System. Lyon: IARC Press, 2000, pp 176-189

29. Mar JC, Wells CA, Quackenbush J: Defining an informativeness metric for clustering gene expression data. Bioinformatics 27:1094-1100, 2011

30. Meester-Smoor MA, Janssen MJ, Grosveld GC, de Klein A, van IJcken WF, Douben H, et al: MN1 affects expression of genes involved in hematopoiesis and can enhance as well as inhibit RAR/RXR-induced gene expression. Carcinogenesis 29:2025-2034, 2008

31. Meester-Smoor MA, Molijn AC, Zhao Y, Groen NA, Groffen CA, Boogaard M, et al: The MN1 oncoprotein activates transcription of the IGFBP5 promoter through a CACCC-rich consensus sequence. J Mol Endocrinol 38:113-125, 2007

32. Mumford JA, Horvath S, Oldham MC, Langfelder P, Geschwind DH, Poldrack RA: Detecting network modules in fMRI time series: a weighted network analysis approach. Neuroimage 52:1465-1476, 2010 
33. Obayashi T, Hayashi S, Shibaoka M, Saeki M, Ohta H, Kinoshita K: COXPRESdb: a database of coexpressed gene networks in mammals. Nucleic Acids Res 36 (Database issue):D77D82, 2008

34. Ozaki S, Nishizaki T, Ito H, Sasaki K: Comparative genomic hybridization analysis of genetic alterations associated with malignant progression of meningioma. J Neurooncol 41:167174, 1999

35. Perry A, Gutmann DH, Reifenberger G: Molecular pathogenesis of meningiomas. J Neurooncol 70:183-202, 2004

36. Pfisterer WK, Coons SW, Aboul-Enein F, Hendricks WP, Scheck AC, Preul MC: Implicating chromosomal aberrations with meningioma growth and recurrence: results from FISH and MIB-I analysis of grades I and II meningioma tissue. J Neurooncol 87:43-50, 2008

37. Plun-Favreau H, Perret D, Diveu C, Froger J, Chevalier S, Lelièvre $\mathrm{E}$, et al: Leukemia inhibitory factor (LIF), cardiotrophin-1, and oncostatin $\mathrm{M}$ share structural binding determinants in the immunoglobulin-like domain of LIF receptor. $\mathbf{J}$ Biol Chem 278:27169-27179, 2003

38. Presson AP, Yoon NK, Bagryanova L, Mah V, Alavi M, Maresh EL, et al: Protein expression based multimarker analysis of breast cancer samples. BMC Cancer 11:230, 2011

39. Ragel BT, Jensen RL: Aberrant signaling pathways in meningiomas. J Neurooncol 99:315-324, 2010

40. Ragel BT, Jensen RL: Molecular genetics of meningiomas. Neurosurg Focus 19(5):E9, 2005

41. Ransom CB, Liu X, Sontheimer H: BK channels in human glioma cells have enhanced calcium sensitivity. Glia 38:281291, 2002

42. Ravasz E, Somera AL, Mongru DA, Oltvai ZN, Barabási AL: Hierarchical organization of modularity in metabolic networks. Science 297:1551-1555, 2002

43. Riemenschneider MJ, Perry A, Reifenberger G: Histological classification and molecular genetics of meningiomas. Lancet Neurol 5:1045-1054, 2006

44. Shannon P, Markiel A, Ozier O, Baliga NS, Wang JT, Ramage D, et al: Cytoscape: a software environment for integrated models of biomolecular interaction networks. Genome Res 13:2498-2504, 2003

45. Sharan R, Ideker T: Modeling cellular machinery through biological network comparison. Nat Biotechnol 24:427-433, 2006

46. Shimon I, Yan X, Magoffin DA, Friedman TC, Melmed S: Intact leptin receptor is selectively expressed in human fetal pituitary and pituitary adenomas and signals human fetal pituitary growth hormone secretion. J Clin Endocrinol Metab 83:4059-4064, 1998

47. Smyth GK: Linear models and empirical bayes methods for assessing differential expression in microarray experiments. Stat Appl Genet Mol Biol 3:Article3, 2004

48. Vidal M, Cusick ME, Barabási AL: Interactome networks and human disease. Cell 144:986-998, 2011

49. Wang H, Wang H, Zhang W, Fuller GN: Overexpression of IGFBP5, but not IGFBP3, correlates with the histologic grade of human diffuse glioma: a tissue microarray and immunohistochemical study. Technol Cancer Res Treat 5:195-199, 2006
50. Wang K, Li M, Hadley D, Liu R, Glessner J, Grant SF, et al: PennCNV: an integrated hidden Markov model designed for high-resolution copy number variation detection in wholegenome SNP genotyping data. Genome Res 17:1665-1674, 2007

51. Wilson CB: Meningiomas: genetics, malignancy, and the role of radiation in induction and treatment. The Richard C. Schneider Lecture. J Neurosurg 81:666-675, 1994

52. Wöhrle FU, Daly RJ, Brummer T: Function, regulation and pathological roles of the Gab/DOS docking proteins. Cell Commun Signal 7:22, 2009

53. Wrobel G, Roerig P, Kokocinski F, Neben K, Hahn M, Reifenberger G, et al: Microarray-based gene expression profiling of benign, atypical and anaplastic meningiomas identifies novel genes associated with meningioma progression. Int J Cancer 114:249-256, 2005

54. Wu J, Lingrel JB: KLF2 inhibits Jurkat T leukemia cell growth via upregulation of cyclin-dependent kinase inhibitor p21WAF1/CIP1. Oncogene 23:8088-8096, 2004

55. Xiang Y, Zhang CQ, Huang K: Predicting glioblastoma prognosis networks using weighted gene co-expression network analysis on TCGA data. BMC Bioinformatics 13 Suppl 2: S12, 2012

56. Yau C, Mouradov D, Jorissen RN, Colella S, Mirza G, Steers $\mathrm{G}$, et al: A statistical approach for detecting genomic aberrations in heterogeneous tumor samples from single nucleotide polymorphism genotyping data. Genome Biol 11:R92, 2010

57. Zhang B, Horvath S: A general framework for weighted gene co-expression network analysis. Stat Appl Genet Mol Biol 4:Article17, 2005

58. Zhu XL, Ai ZH, Wang J, Xu YL, Teng YC: Weighted gene co-expression network analysis in identification of endometrial cancer prognosis markers. Asian Pac J Cancer Prev 13: 4607-4611, 2012

Manuscript submitted August 10, 2013.

Accepted October 1, 2013.

Please include this information when citing this paper: DOI: 10.3171/2013.10.FOCUS13326.

Address correspondence to: William J. Mack, M.D., Department of Neurosurgery, Keck School of Medicine, University of Southern California, Los Angeles, 1200 N. State St., Ste. 3300, Los Angeles, CA 90033. email: mackw@usc.edu.

Supplemental online information: Supplementary tables are hosted on the file server in Dr. Kai Wang's lab at the University of Southern California, Los Angeles.

Supplementary Table 1 ("Expression signal value and copy number of differentially expressed genes"): http://bionas.usc.edu/meningioma/ Table_S1.xlsx.

Supplementary Table 2 ("Comparison between 3 gene expression data sets"): http://bionas.usc.edu/meningioma/Table_S2.xlsx.

Supplementary Table 3 ("Genes with high correlation between copy number and expression level"): http://bionas.usc.edu/meningioma/ Table_S3.xlsx.

Supplementary Table 4 ("Aberrant signaling pathways in meningiomas"): http://bionas.usc.edu/meningioma/Table_S4.xlsx. 